Check for updates

Cite this: Mater. Chem. Front., 2021, 5, 3788

Received 12th November 2020,

Accepted 8th February 2021

DOI: 10.1039/d0qm00922a

rsc.li/frontiers-materials

\section{Photodynamic therapy: photosensitizers and nanostructures}

\author{
Alberto Escudero, (D)*ab Carolina Carrillo-Carrión, (D) ${ }^{c} M^{a}$ Carmen Castillejos, (D) ${ }^{b}$ \\ Elena Romero-Ben, (iD ${ }^{b}$ Christian Rosales-Barrios (iD ${ }^{b}$ and Noureddine Khiar (iD *b
}

${ }^{a}$ Departamento de Química Inorgánica, Facultad de Química, Universidad de Sevilla, Calle Profesor García González 1, E-41012 Seville, Spain. E-mail: alberto.escudero@csic.es

${ }^{b}$ Instituto de Investigaciones Quimicas (IIQ), CSIC-Universidad de Sevilla, Avenida Américo Vespucio 49, E-41092 Seville, Spain

${ }^{c}$ Departamento de Química Orgánica, Universidad de Córdoba, Campus de Rabanales, Edificio Marie Curie, Ctra Nnal IV-A Km. 396, E-14014, Cordoba, Spain

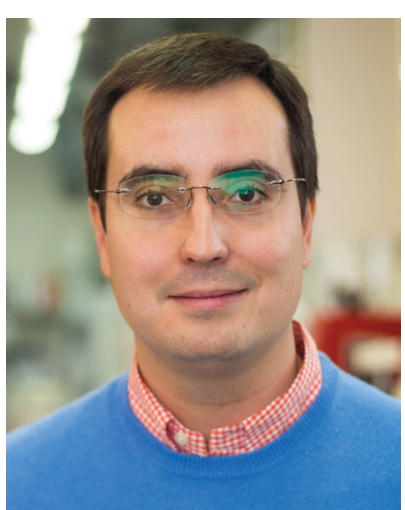

Alberto Escudero
Alberto Escudero obtained his $\mathrm{PhD}$ in Chemistry from the University of Seville in 2007. Afterwards, he was a postdoctoral researcher at the German universities of Jena, Bayreuth, and Marburg, and at the Materials Science Institute of Seville, Spain. He also worked as a researcher for the Leibniz Institute for New Materials in Saarbrücken, Germany. Since 2019, he has been an assistant professor at the Department of Inorganic Chemistry of the University of Seville, Spain, and a researcher at the Institute for Chemical Research (CSIC, University of Seville). His research lines are focused on the synthesis, functionalization and applications of inorganic and hybrid nanomaterials.

\section{Introduction: fundamentals of photodynamic therapy and its physical mechanisms}

Photodynamic therapy (PDT), along with photothermal therapy (PTT) and magnetic hyperthermia, is one of a collection of techniques in medicine that uses the response of sensitive compounds or nanostructures to external stimuli. Under appropriate circumstances, some structures are able to produce reactive oxygen species (ROS) after being excited with light in

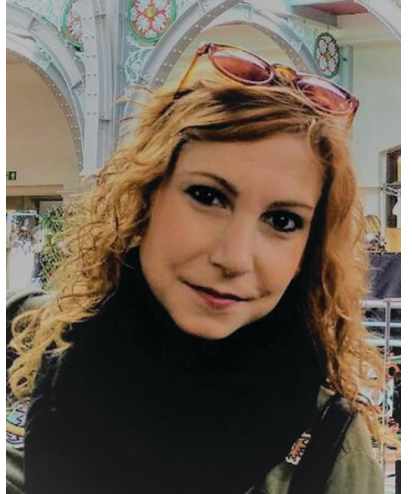

Carolina Carrillo-Carrión
Carolina

Carrillo-Carrión obtained her PhD from the University of Cordoba in 2011. Afterwards, she held postdoctoral postitions at the Philipps-Universität Marburg (Germany), CIC biomaGUNE (Spain) and CiQUS-University of Santiago de Compostela (Spain). In 2020, she joined the University of Cordoba as principal investigator of a project for young researchers. She will join the University of Seville and the Institute for Chemical Research (CSIC, University of Seville) as "Ramón y Cajal" Researcher in April 2021. She has expertise in the synthesis and application of functional hybrid nanomaterials, and is currently working on metal-organic framework-based nanocomposites with applications in biomedicine and catalysis. 
the presence of oxygen. This can be used for the direct killing of cells, either by vascular damage or by activation of an immune response against them. ${ }^{1-3}$

The term photosensitizer (PS) is usually used to refer to such species. Traditionally, some organic dyes ${ }^{4}$ and aromatic hydrocarbons such as xanthene - derivative dyes (Rose Bengal ${ }^{5}$ and $\operatorname{eosin}^{6}$ ) as well as methylene blue ${ }^{7}$ - have been used as PSs. The production of ROS is related to the electronic transitions among the different excited states of the dye, followed by an eventual electron or energy transfer to an oxygen species.

The mechanisms of the production of ROS are closely related to those of luminescence, and are represented in Fig. 1. A photosensitive species can have at least two excited states: a singlet and a triplet. Typical electronic transitions in photosensitive species take place between the ground $\left(\mathrm{S}_{0}\right)$ and the singlet excited states (such as $S_{1}$ or $S_{2}$ ) after the appropriate absorption of radiation, but a further transition to the triplet excited state $\left(\mathrm{T}_{1}\right)$, in principle forbidden, may also occur. ${ }^{8}$
The term intersystem crossing is used to refer to such a phenomenon. An appropriate energy difference between the excited states ${ }^{9}$ and the presence of heavy atoms ${ }^{10}$ in the PS favour this intersystem crossing. Once in the triplet state, two mechanisms may take place: (i) the excited electron may be transferred to other species yielding free radicals that can then react with oxygen to form ROS, such as superoxide radical anions $\left(\mathrm{O}_{2}{ }^{\bullet-}\right), \mathrm{H}_{2} \mathrm{O}_{2}$, and hydroxyl radicals $\left({ }^{\bullet} \mathrm{OH}\right)$, i.e., mechanism type 1 ; and (ii) this energy can be transferred to triplet oxygen $\left({ }^{3} \mathrm{O}_{2}\right)$ to form singlet oxygen $\left({ }^{1} \mathrm{O}_{2}\right)$, i.e., mechanism type $2 .{ }^{11,12}$ For both mechanisms, the more probable the intersystem crossing, the more efficient the production of ROS.

PSs are usually conjugated unsaturated organic molecules, often containing heavy atoms. Some transition metal complexes as well as some semiconductors ${ }^{13}$ and other inorganic nanoparticles and nanostructures ${ }^{14}$ have also been reported. Ideal PSs should possess high light absorption coefficients, ideally at long wavelength radiations (infrared or near-infrared

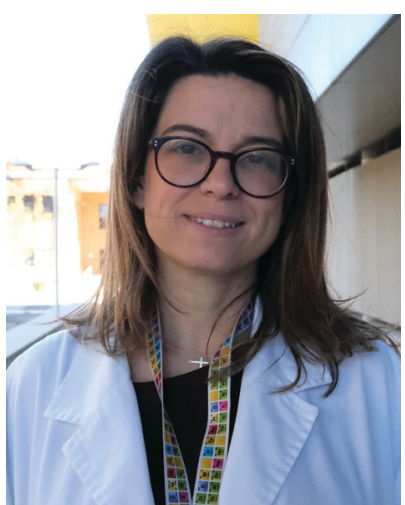

M Carmen Castillejos
$M^{a}$ Carmen Castillejos received her BSc in Pharmacy from the University of Seville, and her MSc in Health Science Current Research Trends from the University of Malaga, Spain. She is currently pursuing her PhD degree in selfassembled nanomaterials for biomedical applications at the Institute for Chemical Research (CSIC, University of Seville) under the supervision of Dr. Noureddine Khiar.

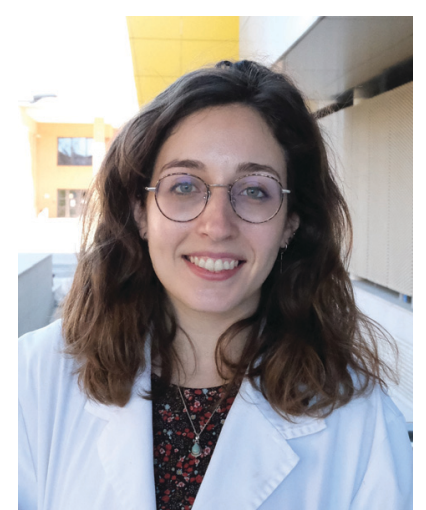

Elena Romero-Ben
Elena Romero-Ben received her BSC in Biology from the University of Seville, Spain, and her MSc in Health Biotechnology from the University Pablo de Olavide. She is currently pursuing her PhD degree in selfassembled nanomaterials for biomedical applications at the Institute for Chemical Research (CSIC, University of Seville) under the supervision of Dr. Noureddine Khiar.

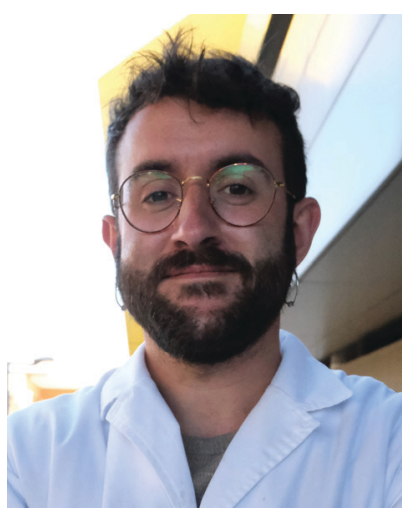

Christian Rosales-Barrios
Christian Manuel RosalesBarrios received his BSc in Biology from the University of Seville, Spain, and his MSc in Health Biotechnology from the University Pablo de Olavide. He is currently pursuing his PhD degree in self-assembled nanomaterials for biomedical applications at the Institute for Chemical Research (CSIC, University of Seville) under the supervision of Dr. Noureddine Khiar.

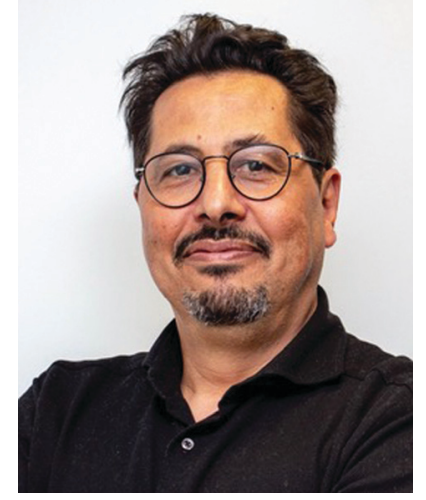

Noureddine Khiar

Noureddine Khiar received a PhD in Organic Chemistry from the University of Strasbourg, France. After several post-doctoral positions in the USA and Europe, in 1997 he joined the newly created Institute of Chemical Research (IIQ, CSIC, University of Seville). $\mathrm{He}$ is currently the Director of the IIQ, Senior Research Scientist of the CSIC, Principal Investigator of the group "Asymmetric Synthesis and Functional Nanosystems", and is responsible for the research group of the Junta de Andalucia "Organic Synthesis and Molecular Recognition". His main scientific interests are the rational design of bionanomaterials and their applications in medicine, and the development of new asymmetric transformations for the synthesis of biologically relevant products. 


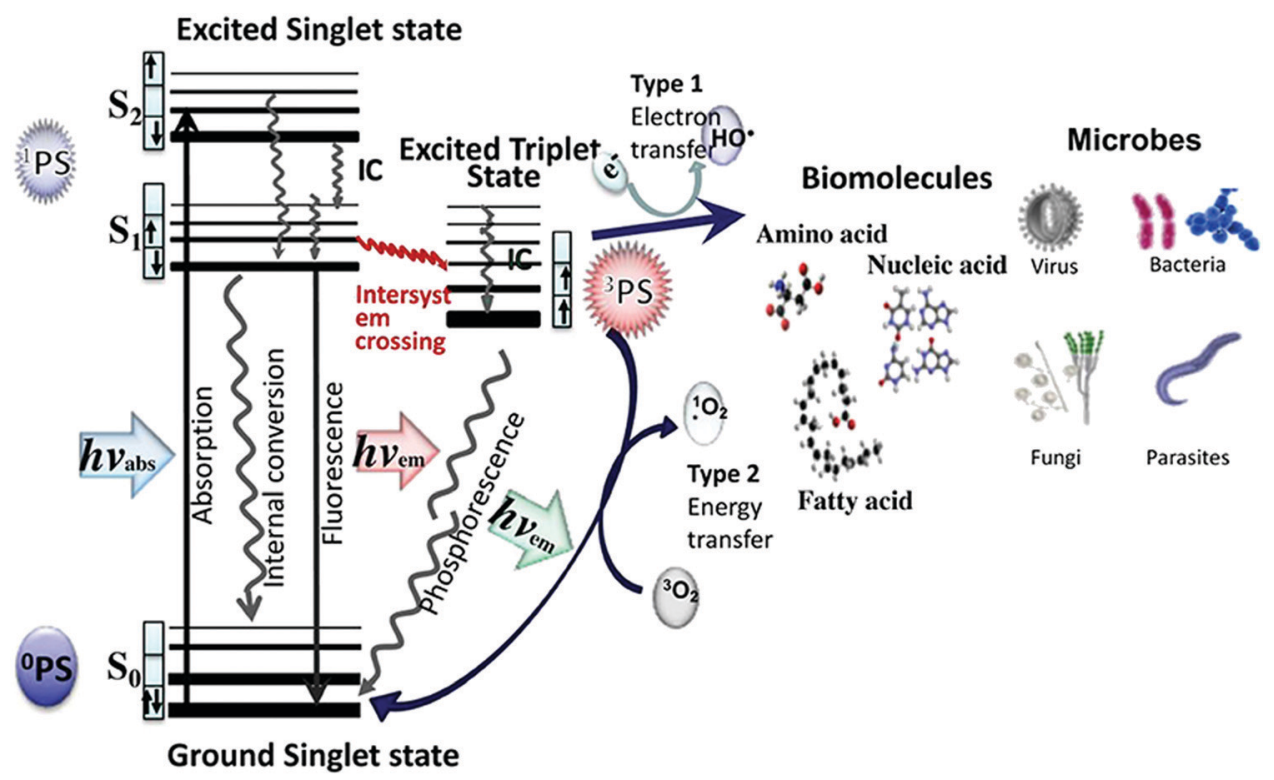

Fig. 1 Electronic states and possible transitions in a photosensitizer, and mechanisms for the formation of radical oxygen species (ROS). Taken from ref. 11, published by Frontiers.

(NIR)), to ensure better tissue penetration, ${ }^{15}$ low photobleaching quantum yields, high intersystem crossing efficiencies, as well as low toxicity in the absence of light, and in principle, an appropriate hydrophilic and hydrophobic balance itself to favour selective accumulation in tumours. ${ }^{16}$

Different structures have been used as PSs. ${ }^{14}$ Porphyrins, with special attention to the hematoporphyrin derivative (HpD), constitute the first-generation PSs, which were started to be used in the 1970s. They are naturally occurring compounds that usually show high quantum yields for the production of ROS, although they might suffer from photobleaching. Other structures such as derivatives of hematoporphyrin and chlorins were designed to overcome some of the limitations of the previously used compounds, such as their lack of solubility, given that most of them are hydrophobic, or to improve their optical properties. They are the second-generation of PSs. As in many other cases, the development of nanotechnology offered new possibilities and implementations. Nanostructures possess the size of $1-100 \mathrm{~nm}$ in which most of the biomolecular interactions take place. ${ }^{17,18}$ In particular, the conjugation or incorporation of PSs into nanostructures paved the way for their use in nanomedicine. The possibility of multifunctionality, including targeting to selectively treat tumour tissues, as well as possible improvements in intracellular delivery in terms of efficiency and selectivity are some of the advantages of the so-called third-generation of PSs. ${ }^{19}$ Different nanostructures such as inorganic nanoparticles (NPs), micelles, and vesicles, especially liposomes, with already developed commercial formulations, have recently been used for the conjugation with PSs. The fourth-generation PSs are related to the use of porous carriers, which include mesoporous silica and metal-organic frameworks (MOFs). These structures can incorporate a large number of sensitizer molecules. From the pharmaceutical point of view, the term pharmaceutical formulation of PSs is also used to refer to both third- and fourth-generation PSs.

This review will summarise the different PS structures that have been proposed to be used for PDT, with a description of their chemistry and physical properties, as well as their incorporation into nanostructures and porous nanomaterials.

\section{Photosensitizers}

\section{2a. Porphyrins, chlorines, purpurins, and phthalocyanines}

Porphyrins are compounds based on a ring of four interconnected modified pyrrole subunits, and are usually hydrophobic. Due to their high level of conjugation, they are well known for absorbing visible light. In some cases, they might also have a metallic atom in the center, resulting in a naturally occurring group of chromoproteins, including hemoglobin and chlorophyll. The most important advantage of such compounds is their relatively high quantum yields $(\Phi)$ for the production of ROS, and their rich chemistry. In fact, the original porphyrin structure can be modified, affecting its optical properties such as the absorbance spectrum. Photobleaching, low absorption in the IR or NIR spectral window and poor selectivity ${ }^{20}$ are their main drawbacks.

Hematoporphyrin derivative (HpD, Fig. 2a) and photofrin II (a purified version of $\mathrm{HpD}$ ) are good examples of these types of PSs, and can be considered as first-generation PSs. In fact, they are nowadays widely used as PSs. ${ }^{21}$ Both structures absorb radiation at around $630 \mathrm{~nm}$, and show a relatively high quantum yield for the production of singlet molecular oxygen $\left(\Phi=0.64\right.$ in the case of $\mathrm{HpD}$, in methanol ${ }^{22}$ ). Both compounds have been used to treat brain, laryngeal, lung, skin, gastric, colorectal, and esophageal carcinomas. ${ }^{23,24}$

Modifications of the structure of porphyrins constitute the so-called second-generation PSs. For example, chlorines are 


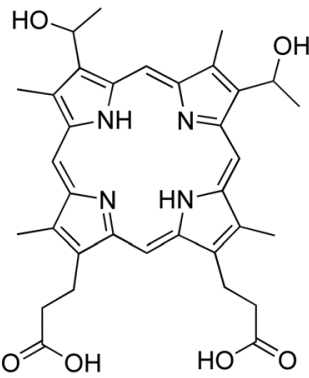

a<smiles>NCC(=O)CCC(=O)O</smiles>

d

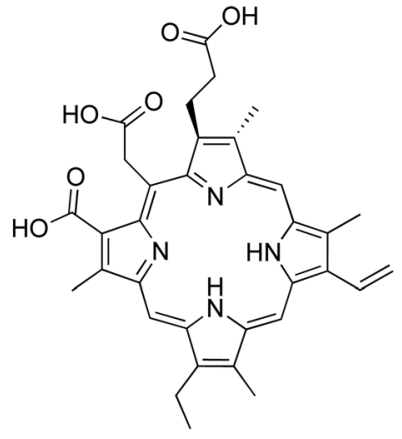

b<smiles></smiles>

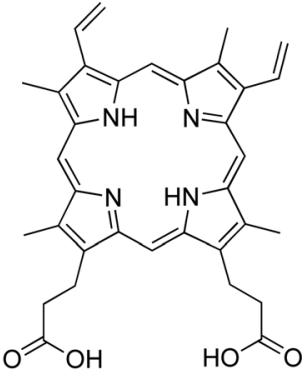

$c$<smiles></smiles>

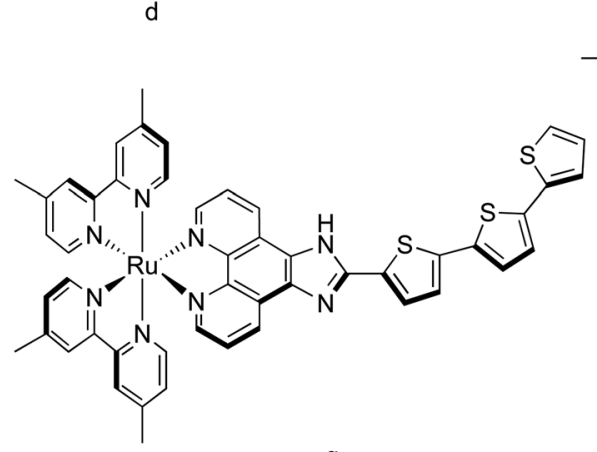

e

f<smiles>N#CC(C#N)=C(/C=C/c1ccc(OC(=O)O[Na])cc1)C1=CCC(c2ccc(C(=C(c3ccccc3)c3ccc(OS(=O)(=O)c4ccc([N+](=O)[O-])cc4[N+](=O)[O-])cc3)c3ccc(OS(=O)(=O)c4ccc([N+](=O)[O-])cc4[N+](=O)[O-])cc3)cc2)=[SH]1</smiles>

$\mathrm{h}$

Fig. 2 Examples of some compounds used as photosensitizers for PDT: (a) hematoporphyrin, (b) chlorin e6, (c) protoporphyrin IX, (d) 5-aminolevulinic acid (AVA), (e) iodine-substituted BODIPY, (f) aza-BODIPY derivative, (g) Ru[(dmb) $\left.{ }_{2}(\mathrm{IP}-3 \mathrm{~T})\right] \mathrm{Cl}_{2}$, and (h) AlEgen-based photosensitizer containing a tetraphenylethylene moiety.

formed after the saturation of one or two double bonds of the porphyrin structure. This shifts the absorbance spectra to longer wavelengths and thus toward the therapeutic window. ${ }^{25}$ Chlorin e6 (Ce6) and its derivatives (hydrophobic, Fig. $2 b)^{26}$ have been used for the treatment of bile duct carcinoma ${ }^{27}$ as well as breast and hypopharyngeal cancer cells. $^{28}$ A chlorin-based compound, termoporfin (m-THPC, hydrophobic, with an absorption maximum at $650 \mathrm{~nm}$ ), is in fact a clinically used $\mathrm{PS}^{29}$ some formulations of which are commercially available. Protoporphyrin IX (PpIX, hydrophobic, Fig. 2 c) ${ }^{30}$ and the 5-aminolevulinic acid (ALA, hydrophilic, the biological precursor of PpIX) ${ }^{31}$ (Fig. 2d) are also examples of this generation of PSs, and have been applied, for example, in malignant glioma surgery. ${ }^{32,33}$ In fact, the use of ALA to drive the production of intracellular PpIX is in common clinical use. $^{34}$ Some studies regarding both dark cytotoxicity and toxicity under light irradiation of different PSs can be found in the literature. For example, for human epidermoid carcinoma cells, ALA-induced PpIX showed both dark toxicity and phototoxicity of 2-3 orders of magnitude lower than those of photofrin, and a higher dark toxicity:phototoxicity ratio, whereas formulations based on m-THPC showed much more toxicity under light irradiation and a much higher dark toxicity: phototoxicity ratio. ${ }^{35}$

Within this PS generation, naphthalocyanines, which bear a second benzene ring, ${ }^{36}$ and purpurins, ${ }^{37}$ based on a chlorophyll derivative, have also been proposed. Most of these PSs are hydrophobic, even when in some cases chemical modifications can be designed to yield hydrophilic derivatives. In general terms, even when their commercial formulations are used to treat different cancers, the synthesis of these PSs as well as the required structural modifications are difficult. For this reason, non-porphyrin photosensitizers that can be made more easily, thus becoming more synthetically accessible, have started to receive research interest.

\section{2b. BODIPY and derivatives}

BODIPY dyes, based on the 4,4-difluoro-4-bora-3a,4a-diaza-sindacene core, have also been suggested to be used as PSs for PDT. In general terms, such compounds show high absorption 
coefficients, low photobleaching, relatively low toxicity in the absence of light, and low influence of the chemical environment on their properties. ${ }^{16}$ However, they possess high luminescence efficiency, meaning that the intersystem crossing transitions generally have a low probability. The consequence of this is that they produce a low level of ROS. This can be improved by modifying their structure, in most of the cases by incorporating heavy atoms. For example, the ROS quantum yield improves up to 50 times when two iodine atoms are introduced in the core structure. ${ }^{38}$ Further modifications of the BODIPY core with the introduction of up to four iodine atoms and a $p$-tolyl hydrocarbon substituent give rise to structures with high absorption coefficients. These species can be excited from 548 to $581 \mathrm{~nm}$ and show quantum yields higher than 0.83 for the production of $\operatorname{ROS}^{39}$ (Fig. 2e). Absorptions shifted to the NIR region can be obtained by using aza-BODIPY derivatives, as is the case of dibromo-aza-BODIPY (known as ADPM06, Fig. 2f), at expenses of slight changes in their absorption coefficients and the ROS quantum yield generation, as well as some aqueous solubility issues. ${ }^{8,40}$ Aza-BODIPY derivatives have been suggested to be closer to clinical development than any other subcategory in their category, and although they are less synthetically accessible, ${ }^{16}$ they currently attract wide research attention. ${ }^{41}$ In general terms, BODIPY-based PSs are hydrophobic, but as in the previously commented species, hydrophilic derivatives can be obtained after appropriate chemical modifications. ${ }^{42}$

\section{2c. Transition metal complexes, aggregation-induced emission species, nanoparticles, and other structures}

Similar quantum yields for the production of ROS than those shown by traditionally used organic dyes have also been reported for some transition metal complexes, as occurs with $\left[\mathrm{Ru}(\mathrm{bpy})_{3}\right]^{2+}$ (excitable at $\left.452 \mathrm{~nm}\right)^{43}$ and $\mathrm{rac}-\mathrm{Ru}\left[(\mathrm{dmb})_{2}\right.$ (IP-3T) $] \mathrm{Cl}_{2}$ (known as TLD1433, Fig. 2g, excitable at $525 \mathrm{~nm}$, and used for the treatment of nonmuscle-invasive bladder cancer). In general terms, transition metal complexes show rich photochemistry, given their variety of excited-state electronic configurations accessible with visible and near-infrared light, which can be designed to enhance both energy- and electrontransfer processes. Other metal complexes of $\mathrm{Pt}(\mathrm{Iv}), \mathrm{Ru}(\mathrm{II}), \mathrm{Rh}(\mathrm{III})$, Ir(III) and Os(II) have also been proposed. ${ }^{44}$ Rhenium(I) carbonyl complexes with ligands such as bpy, phen, pyridazine derivatives and BODIPY have also been reported and used, reaching high quantum yields for the production of ROS. ${ }^{45}$

Aggregation-induced emission (AIE) species, also known as AIE luminogens or AIEgens, have recently emerged as a new class of luminescent materials with applications in biosensing. Such species show an enhanced luminescence upon aggregate formation, contrary to what usually happens with conventional organic dyes, in which the fluorescence is weakened or quenched in concentrated solution or in the aggregated state, ${ }^{46}$ and might suffer from attenuated fluorescence and insufficient ROS generation. ${ }^{47}$ This phenomenon was first observed in molecules with twisted structures and freely rotatable peripheral aromatic moieties, as is the case of hexaphenylsilole, ${ }^{48}$ tetraphenylethene, diphenylfumaronitrile and distyreneanthracene, ${ }^{49}$ and it has been associated with the restriction of intramolecular motions, rotations and vibrations within the molecule when they are in an aggregated state. ${ }^{50}$ AIEgens are used in the development of fluorescent biosensors with superior sensitivity, but in some cases, their molecular structures can be designed to show both high fluorescence and efficient ROS generation upon aggregation..$^{51}$ Taking into account that most of the PSs show a hydrophobic character, and they thus tend to aggregate in aqueous media, AIEgens are currently attractive PSs, receiving research attention and having been proposed for image-guided PDT. $^{52-54}$ An AIEgen-based PS containing a tetraphenylethylene moiety is shown in Fig. $2 \mathrm{~h}$.

Nanoparticles (NPs) can also be used in PDT, not only as a functional carrier, as will be discussed in the following section, but also as a ROS source or for the modulation of the production of other species. ${ }^{55,56}$ In general terms, they offer a wider possibility of excitation, ranging from ultraviolet to visible, infra-red and even X-rays, as well as higher chemical stability, when compared to that of their molecular counterparts. Nanoparticles with uses in PDT can be classified into three categories, as follows: (1) NPs capable of producing ROS by themselves. This is the case of fullerenes and their derivatives. Such structures have an extended $\pi$-conjugation which allows them to absorb visible light, and also show a high triplet yield that can thus generate reactive oxygen species upon illumination. ${ }^{57}$ Their main drawback is that they are highly hydrophobic and consequently are prone to aggregates, thus requiring appropriate functionalization or encapsulation strategies for their use..$^{58}$ (2) NPs consisting of an absorbing semiconducting polymer, which can act as a PS, as is the case of PCPDTBT, ${ }^{59}$ and other polymer-based structures. ${ }^{60}$ (3) Nanostructures composed of well-known photoactive materials such as titanium dioxide $\left(\mathrm{TiO}_{2}\right)$ have also been suggested, ${ }^{61}$ although the fact that they absorb in the UV region of the spectra limits their use in the treatment of superficial tumours. ${ }^{62}$ Core-shell nanostructures based on titaniacoated upconversion nanoparticles (UCNPs) have been recently proposed to overcome the limitation of the UV excitation requirements of $\mathrm{TiO}_{2}$-based structures. ${ }^{63,64}$ UCNPs are able to absorb NIR radiations and emit visible and UV light, which excite $\mathrm{TiO}_{2}$. A similar strategy has been used for graphene quantum dots decorated with UCNPs. ${ }^{65}$ This concept introduces the second group of NP-based PSs, in which the inorganic NPs absorb radiations and transfer them to the active PS species, including possible FRET processes. ${ }^{66,67} \mathrm{CdSe} / \mathrm{CdS} / \mathrm{ZnS}$ quantum dots conjugated with organic dyes such as Rose Bengal or chlorin $e 6^{68}$ are representative examples of nanostructures also belonging to this category. Some inorganic silicate-based NPs have also been proposed as X-ray excitable nanosensitizers, combining the advantages of both radiotherapy and PDT. ${ }^{69}$ Finally, some nanostructures can also act as quenchers of the luminescence of active PSs, thus enhancing the possibilities of non-radiative relaxation and then the efficiency of the production of ROS. This is the case, for example, of carbon nanotubes, which have been used to improve the ROS efficiency production in chlorin by quenching its luminescence. ${ }^{70}$ 


\section{Incorporation into nanostructures}

The third- and fourth-generation PSs are those in which the active optical species is incorporated into a nano or porous structure. This is a key point for the eventual biomedical applications of such structures, given that nanostructures possess the size in which most of the biological interactions take place. ${ }^{17}$ Specifically, to prevent fast renal clearance from the circulation and non-specific permeation across fenestrated sinusoids of the lymphoid, liver, bone marrow or spleen, the size of the NP carriers must be $>20 \mathrm{~nm} .{ }^{71}$ However, NPs of about 100-200 nm may pass through the pores of the vessels in the tumor microenvironment. ${ }^{72}$ Thus, NPs within a size range of 20-50 $\mathrm{nm}$ would be a better choice. Moreover, in terms of delivery, it should be noted that many drugs suffer from poor solubility and stability, ${ }^{73}$ especially most of the PSs. Thus, their incorporation into nanostructured carriers usually improves the delivery efficiency, ${ }^{74,75}$ and can also allow a spatiotemporally controlled release of the drug in response to specific stimuli. ${ }^{76}$

Gold, silica, iron oxide and upconverting nanoparticles (UCNPs) are inorganic NPs widely used in biomedicine, and many examples of the incorporation of PSs into such structures can be found in the literature. Polymer-based NPs have also been described, with a special interest in natural biodegradable polymers such as alginate and chitosan, and other carbohydrate and protein derivatives. Amphiphilic structures forming micelles and vesicles are also widely used as carriers for PDT, as well as nanosized metal-organic frameworks (MOFs), which have been proposed to carry diverse PSs, either by encapsulation of the PSs into the pores of the MOFs or by the integration of the PSs as building units of the framework structure.

Depending on the PS nature, different strategies for the attachment/conjugation to the carriers have been reported, including encapsulation, covalent conjugation, physical adsorption, and hydrophobic interactions, among others. ${ }^{55}$

The advantages and disadvantages of the incorporation of PSs into both inorganic and organic nanostructures are related to the properties or characteristics of the nanostructures themselves. On one hand, inorganic nanostructures can provide the systems with additional functionalities, as is the case of magnetism and luminescence. Moreover, their physicochemical properties are more dependent on the nature and size of the material, which are easily modified in most of the NP types, providing a better control of their properties. ${ }^{77}$ On the other hand, organic self-assembled nanostructures such as liposomes are especially appropriate for the encapsulation of hydrophobic cargos, and thus for drug delivery applications. ${ }^{78}$ The upscaling and the large-scale production of organic nanostructures are much more developed, which also explain the commercial availability of many liposome-based formulations. Another important point to consider is the toxicity. In general terms, inorganic nanostructures usually show higher toxicity levels than their organic counterparts, which in many cases can also be biodegradable. This can somehow limit their uses for in vivo applications. ${ }^{79}$

\section{3a. Inorganic nanostructures}

Gold nanoparticles have been widely studied in the last few decades, and are probably the most widely used inorganic nanoparticles in nanomedicine, being of special interest for delivery applications, among others. ${ }^{80}$ This is in fact the most important role played by Au NPs within PDT, which in most of the cases act as a passive carrier and are used to deliver photosensitizer agents for the PDT of cancer. In some cases, the production of ROS with gold nanostructures themselves has been reported, even when the mechanisms are not fully understood and are currently an open topic. ${ }^{81-83}$

PSs can be incorporated into gold nanostructures either by non-covalent interactions (based on ionic, electrostatic or hydrophobic forces) or by forming covalent bonds with the $\mathrm{Au}$ surface, usually using thiolated linkers. The formation of $\mathrm{N}$-bonds with the Au surface has also been described. ${ }^{84}$ One of the strategies reported in the literature consists of a previous modification of the PS by adding thiol groups to its structure. Given the high affinity of sulfur for metallic gold, Au NPs can be easily functionalised with such species. This is the case of $\mathrm{Au}$ NPs functionalised with $\mathrm{Zn}$ (II)-phthalocyanine, in which the Au NPs are synthesised by following a relatively standard strategy consisting of the reduction of gold precursor in THF in the presence of $\mathrm{Zn}$ (II)-phthalocyanine disulfide (C11Pc), which is eventually linked to the gold surface NPs by forming an $\mathrm{Au}-\mathrm{S}$ bond. The presence of PEG-containing thiol groups, which also link to the Au NPs, has been shown to enhance the solubility of the nanostructure and also to improve the efficiency of the PDT $^{85}$ (Fig. 3a). This strategy has also been reported for PSs such as thiolated palladium tetrapyrrole complexes. ${ }^{86}$ Although much weaker, the incorporation of PSs through the formation of an $\mathrm{Au}-\mathrm{N}$ bond has also been reported. As an example, the terminal amine of a silicon phthalocyanine 4 (Pc4) can be entrapped into PEG-functionalised Au NPs, giving rise to a kind of entrapped structure which the authors define as a "cage-type structure", that can be used to encapsulate hydrophobic PSs. ${ }^{87}$ However, given the weak $\mathrm{Au}-\mathrm{N}$ bond, other stabilising ligands, such as PEG derivatives, are usually demanded.

Typical functionalization of the already synthesised Au NPs has also been reported, as is the case of the conjugation of thiolated PEG-Au nanostars with chlorin e6 through the widely used EDC chemistry. ${ }^{88}$ The same strategy can be used with other linkers or gold nanoparticle shapes, as occurs with glutathione-functionalised Au nanostars further linked with chlorin e6, by using a similar chemistry. ${ }^{89}$ Other PSs such as indocyanine green (ICG) can be electrostatically bonded to gold-bearing nanostructures, for example, Au nanorods/MoS nanoagents, ${ }^{90}$ or porous $\mathrm{Au} @ \mathrm{Rh}$ nanostructures. The latter constitutes one example of the fourth-generation $\mathrm{PSs}^{91}$ (Fig. 3b). Zn porphyrins can also be electrostatically attached to $\mathrm{Au}$ NPs functionalised with amphiphile pyridinium salts by following a similar strategy. ${ }^{92}$

Under appropriate circumstances, Au NPs can play a relatively active role in PDT, even when in some cases, as previously commented, they have been claimed to produce ROS without a PS. Au NPs themselves can, in some cases, modify the ROS 

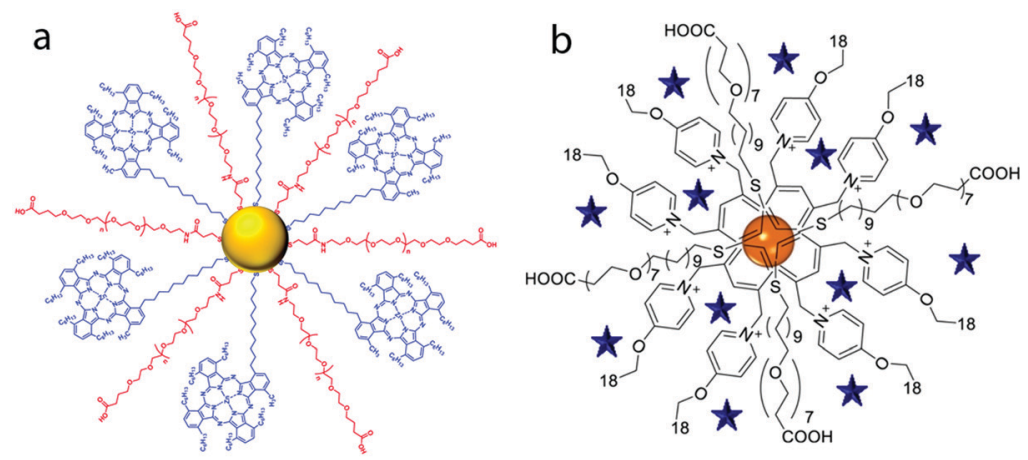

C

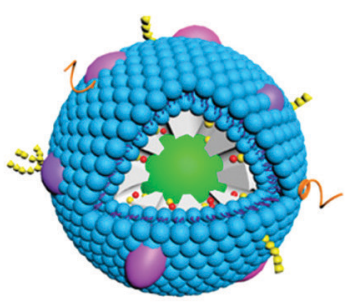

Fig. 3 (a) A phthalocyanine derivative (blue ligand) incorporated into PEG-functionalised Au NPs. Taken from ref. 85. Reproduced with permission from the Royal Society of Chemistry, Copyright (C)2016; (b) pyridinium-stabilised gold nanoparticles with an incorporated anionic Zn(॥) porphyrin (blue stars). Taken from ref. 92. Reproduced with permission from Elsevier, Copyright (C) 2017; (c) silica-coated UCNP. The PSs zinc phthalocyanine (ZnPC) and merocyanine 540 are incorporated into the mesoporous silica coating. An additional coating with a stem-cell membrane provides the nanostructure with long circulation and tumour-targeting capability. Taken from ref. 105. Reproduced with permission from the American Chemical Society, Copyright $(2016$.

production efficiency of active species, given that their fluorescence can be enhanced or quenched due to their proximity to the $\mathrm{Au}$ NP surface. The metal-enhanced fluorescence (MEF) usually occurs for fluorophores located at $c a$. 5-30 nm from the metal surface, while quenching effects in PS-functionalised Au NPs in which the fluorophore is on or very close to the metal surface give rise to metal-enhanced singlet oxygen production $\left(\mathrm{ME}^{1} \mathrm{O}_{2}\right){ }^{93}$ The latter leads to an enhanced PDT effect. This is, for example, the case of Au NPs functionalised with short carbon chain zinc phthalocyanines, which showed higher ROS efficiency production when compared with that of their long carbon chain counterparts. ${ }^{94}$ Another advantage of the use of PS-functionalised Au NPs is their possible applications in multimodal therapies, given that under appropriate conditions, $\mathrm{Au}$ NPs themselves can also be employed for PTT, and thus produce heat for killing cancer cells. This has been reported, for example, for an Au nanoechinus structure, which can be used to treat tumours in mice by both PDT and PTT under NIR irradiation, ${ }^{81}$ and in porphyrin-coated gold nanoshells deposited on silica spheres. ${ }^{95} \mathrm{~A}$ double chemo- and photodynamic therapy has also been proposed for doxorubicin- and chlorin e6-functionalised gold nanoclusters. ${ }^{96}$

The use of other inorganic nanostructures has also been reported in the literature. Silica derivative-based nanostructures are also used for PDT. ${ }^{97}$ In general terms, silica nanostructures are well known for their biocompatibility and the possibility of subsequent functionalization, as well as for the usually high colloidal stability of the final products. ${ }^{98}$ Two main groups of nanostructures can be mostly found in the literature. On one hand, the incorporation of PSs into mesoporous silica-based materials is of special interest, and this constitutes one type of the so-called fourth-generation PSs. On the other hand, silicaderived structures are often used as a coating material of other nanostructures. One example of the first case is silica particles loaded with the photosensitizer Ce6. The most important advantage of such structures is the possibility to incorporate other active species, such as radionuclides, to activate the PS. ${ }^{99}$ The rich chemistry of these materials, including the possibility of layer-by-layer deposition of different compounds, can also be used to design multifunctional carriers that can be used for both PDT and chemotherapy. These structures can also show luminescence properties due to the incorporation of rare-earth cations ${ }^{100}$ or lanthanide upconversion nanoparticles. ${ }^{101}$ Examples of silica particles functionalised with polymers containing the active PS can also be found in the literature. ${ }^{102}$ Examples of the use of silica as a coating material of other nanostructures are the porphyrin-containing silica coating of Au nanorods, ${ }^{103}$ and upconverting nanoparticles coated with mesoporous silica, where PSs can be loaded in the porous matrix, as well as targeting moieties and other functional inorganic NPs can be attached to the silica surface ${ }^{104,105}$ (Fig. 3c). Other mesoporous nanostructures with uses in PDT have also been reported. Some recent examples include X-ray induced deep-penetrating PDT mesoporous silicate nanosensitizers, ${ }^{69}$ mesoporous Ag-embedded $\mathrm{ZnO}$ nanocomposites, ${ }^{106}$ mesoporous ruthenium ${ }^{107}$ and gadolinium orthoniobate $^{108}$ NPs, and mesoporous cerium oxide-coated upconversion nanoparticles. ${ }^{109}$ These nanoplatforms are often used for both chemo- and photodynamic therapies, and in some cases also for bioimaging.

Iron oxide nanoparticles (IONPs) are also often used in nanomedicine given their ability to reach the desired site of treatment upon application of an external magnetic field, also known as magnetic targeting, and their relatively good biocompatibility. Moreover, their possibility of generating heat upon alternating magnetic field stimulation makes them suitable for multiple therapies of tumours, including magnetic hyperthermia. ${ }^{110}$ Different examples of IONPs functionalised with PSs can be found in the literature. ${ }^{111-114}$ Iron oxide NPs can also be incorporated into different nanostructures to couple photodynamic therapy (PDT) to magnetic hyperthermia (MHT) and thus to obtain multifunctionality. One example is liposomes that combine both such magnetic nanoparticles and a photosensitizer, in which the IONPs are introduced into the liposome cavity via a water-in-oil emulsion strategy, and the hydrophobic photosensitizer m-THPC is eventually enclosed in the lipid bilayer. ${ }^{115}$ Combination with other materials such as 
$\mathrm{SiO}_{2}$ and rare earth-based NPs to create more complex nanostructures has also been reported in the literature. ${ }^{116}$

\section{3b. Organic nanostructures}

Capsules, micelles and vesicles are structures widely used for the delivery of many drugs and molecules of biomedical interest, and also have applications in PDT.

Polyelectrolyte-based capsules have also been used for the encapsulation of PSs. These structures are usually synthesised by following the layer-by-layer (LbL) strategy, which involves the alternate deposition of layers of polyelectrolytes of opposite charge on an inorganic core, which is eventually dissolved. ${ }^{117}$ Given that in most of the cases the PSs are relatively small molecules, they are usually incorporated by incubation with previously synthesised polyelectrolyte-based capsules (postloading strategy), as in the case of a water-soluble PS zinc phthalocyanine choline derivative, which can be incorporated into polyelectrolyte capsules by incubation at both room temperature and at $80{ }^{\circ} \mathrm{C} .{ }^{118}$ Hydrophilic PSs can also be incorporated into the capsule wall during the layer-by-layer process, as in the case of silicon(Iv) phthalocyanine. ${ }^{119}$ However, hydrophobic PSs are preferably incorporated into polyelectrolytebased capsules during the inorganic core precipitation (preloading strategy). One example of this is Hypocrellin B (HB)-loaded polyelectrolyte capsules. ${ }^{120}$

Micelles are spherical, ellipsoid, cylindrical, or unilamellar nanostructures, mostly formed by amphiphilic molecules in an aqueous solution that self-assemble into a structure containing a hydrophobic core and a hydrophilic corona. The size of a typical micelle ranges from 5 to $100 \mathrm{~nm} .{ }^{121}$ Polymeric micelles are emerging as attractive drug delivery systems ${ }^{122}$ and are usually used to carry hydrophobic drugs, which are contained in their hydrophobic inner core. Micelles and other selfassembled structures based on low molecular weight amphiphiles are also attracting research interest due to the possibility of a more controlled supramolecular structural design, ${ }^{123-125}$ at the cost of possible stability issues, ${ }^{126}$ for example, toward dilution. ${ }^{127}$ Additional polymerization processes ${ }^{128}$ on such self-assembled nanosystems provide them with enhanced stability, thus making them suitable for delivery applications. ${ }^{129,130}$ Given that many PSs are hydrophobic, micelles are ideal carriers to be used for PDT. ${ }^{131-133}$

Micelles can also be functionalised with additional moieties providing them with new functionalities, especially with targeting molecules. However, the biggest disadvantage of these nanostructures is their lack of stability in the physiological environment, given that they may dissociate and thus provoke premature drug release, thus limiting their efficacy as a drug carrier. ${ }^{134}$ Several strategies such as the use of cross-linking structures, production of polymeric prodrug micelles, and design of micelles with lower critical micelle concentration (CMC) or even unimolecular micelles have been proposed to overcome such limitations. ${ }^{130}$

Two main strategies to introduce the PS into micelles can be found in the literature. On one hand, the PS can be incorporated into the amphiphile, and eventually form the micelles after self-assembly. On the other hand, the micelles can be first prepared by following standard methods, and the PS can be later incorporated or loaded into the structure. Regarding the first group of examples, a common protocol is to use a hydrophobic PS such as porphyrin as the hydrophobic block of the amphiphile, and conjugate it with hydrophilic polymers, yielding PS-containing micelles after their self-assembly in water. ${ }^{133}$ This strategy can be used with other PSs, as in the case of hydrophobic iridium(III) complexes that are conjugated to hydrophilic PEG, giving rise to an amphiphilic block copolymer, and eventually to micelles. ${ }^{135}$ A slight variation of this strategy is the conjugation of the hydrophobic PS with large dendritic wedgets that are supposed to sterically prevent or weaken the aggregation of the center dye molecules, and a subsequent conjugation with hydrophilic polymers such as PEG to eventually form micelles after self-assembly. ${ }^{136}$

Hydrophobic PSs can also be linked to the hydrophobic tail of amphiphilic block copolymers. This has been reported for a porphyrin, which was linked to the polycaprolactone (PCL) part of a PCL-PEG copolymer via an acetal group. This type of porphyrin-based micelles can also be loaded with anti-cancer drugs such as hydrophobic doxorubicin (DOX) (Fig. 4). For such nanostructures, PDT reinforces the delivery of anticancer drugs, given that the formed ROS under irradiation induces the breakdown of endosome and lysosome membranes, and this improves the drug release and facilitates the drug translocation into the cell nucleus. ${ }^{137}$ These multifunctional systems combine chemotherapy with PDT and are proposed as a potential vehicle for overcoming drug resistance in chemotherapy. Examples for loading with more than one anticancer drug can also be found in the literature. ${ }^{138}$

In some cases, the PSs can also be linked to the hydrophilic part of an amphiphilic molecule by following the appropriate chemistry. For example, the porphyrin derivative monoaminoporphyrin can be linked to the hydrophilic segment of the poly(2-aminoethyl methacrylate) (PAEMA)-PCL diblock copolymer. In this case, given that the porphyrin moiety was connected to the hydrophilic part of the polymer, its aggregation in aqueous media could be avoided, and thus the quantum yield and effective energy absorption could be improved, which resulted in the improved photodynamic efficacy. ${ }^{139}$ In other

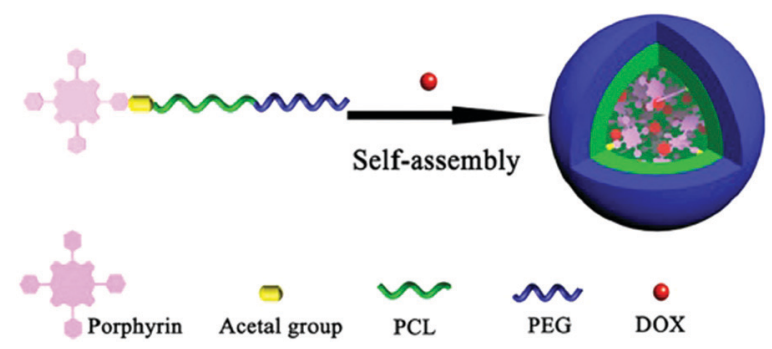

Fig. 4 Example of a micelle formed from an amphiphilic molecule, in which a porphyrin has been linked to the hydrophobic tail (PCL) of an amphiphilic block copolymer. The anticancer drug DOX has also been incorporated. Taken from ref. 137. Reproduced with permission from the American Chemical Society, Copyright (C)2017. 
cases, porphyrins can act as a crosslinker within an amphiphilic block copolymer, increasing the stability of the formed micelles. ${ }^{140}$

Structures related to micelles and based on the self-assembly of hydrophobic polymers with hydrophilic carbohydrate derivatives have also been reported, as in the case of poly(3-hexylthiophene-2,5diyl) (P3HT)-poly(styrene-co-maleic anhydride) (PSMA) core glycoshell nanodots decorated with poly(styrene-co-maleic anhydride) (PSMA), in which the sugars are used as targeting moieties, and the $\mathrm{P} 3 \mathrm{HT}$ is responsible for producing ROS species under irradiation. $^{141}$

The second group of strategies consists of the incorporation of the PS into already formed micelles. In these cases, the result is the photosensitizer is encapsulated and stabilised in aqueous media inside the micelle. ${ }^{142,143}$ In other studies, the photosensitizer can be encapsulated in the micelles during the same micelle formation process. ${ }^{144}$

Liposomes are quite biocompatible round bubbles consisting of an aqueous core encapsulated by natural or synthetic phospholipids. They are one of the most advanced delivery vehicles currently used in nanomedicine, with several formulations approved by the U.S. Food and Drug Administration (FDA). ${ }^{145}$ This is reflected in the high number of studies and formulations that can be found in the literature. Their structure makes them suitable for the encapsulation of both hydrophilic and hydrophobic drugs, which can be incorporated into the core or within the lipid bilayers, respectively. Liposomes are quite advantageous in terms of biocompatibility and biodegradability, although the first-generation liposomes usually suffered from high-cost production, stability in different media, relatively low encapsulation efficiency, and short plasma half-life. ${ }^{146}$ Physicochemical parameters such as the liposome size, surface charge, steric stabilization, membrane lipid packing or route administration can play a key role in the pharmacokinetics of the liposomes. ${ }^{147}$

Given that liposomes are especially suitable for the encapsulation of hydrophobic drugs and that porphyrin-related PSs are usually highly lipophilic, ${ }^{148}$ examples of the encapsulation of PSs for PDT in liposomes have been reported since the last few decades of the last century. ${ }^{149-151}$ Some of these formulations are even currently available as commercial products. For the loading of liposomes, two main loading strategies can be found in the literature. In most of the cases, the typical general synthesis consists of the mixing of the desired phospholipids in the presence of the hydrophobic cargo or PS (passive loading), and a subsequent drying step to form a lipid film. The hydrophobic PS is then entrapped into the lipid bilayer. Liposomes are eventually formed after treatments with aqueous suspensions, which may also contain hydrophilic cargos. ${ }^{152}$ One example of this kind of strategy is the encapsulation of the protoporphyrin IX. In this case, an oleylamine-conjugated protoporphyrin is dissolved in chloroform in the presence of a phospholipid, obtaining the liposomes with the PS entrapped into the lipid bilayer after drying and hydration (Fig. 5A). For this system, the conjugation of the PS with oleylamine moieties enhanced the cell membrane interactions of the porphyrin macrocycles, caused more apoptotic
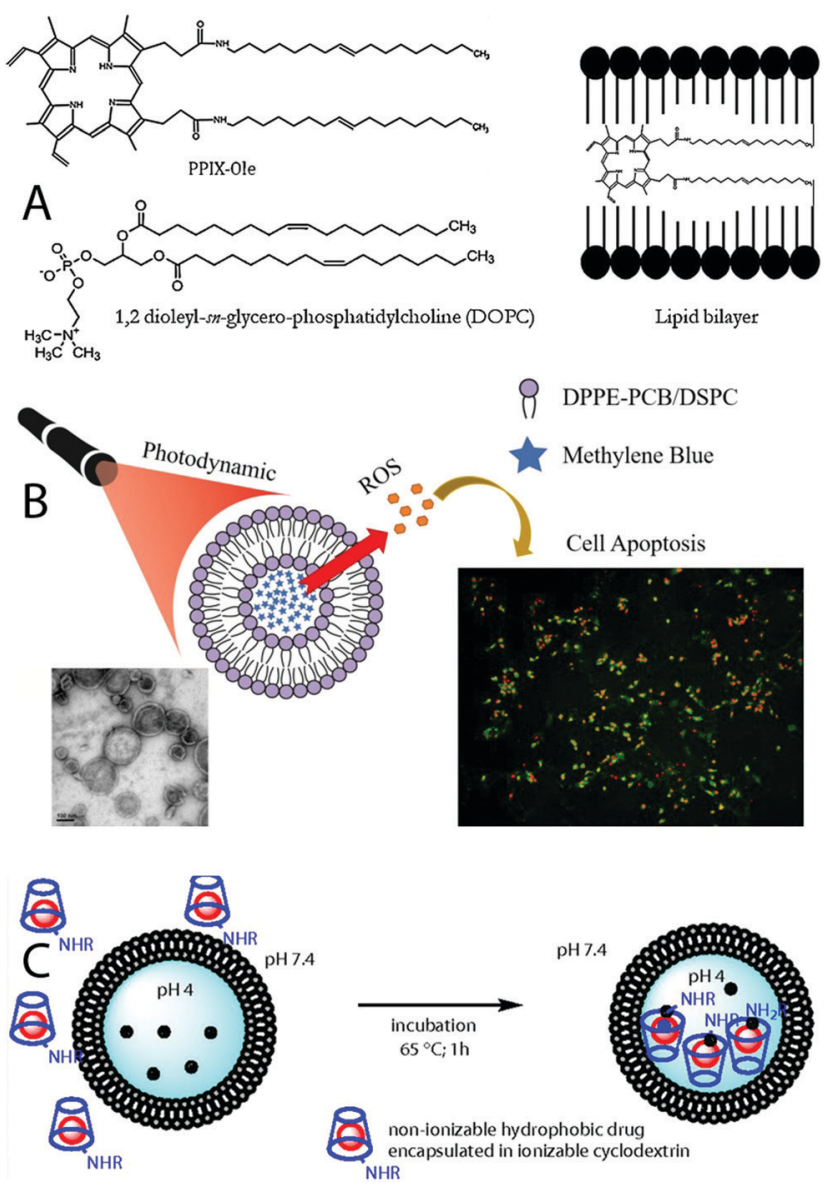

Fig. 5 (A) Encapsulation of a hydrophobic protoporphyrin IX derivative into the lipid bilayer of a liposome. Taken from ref. 153. Reproduced with permission from Elsevier, Copyright $(\mathrm{C} 2014$; (B) encapsulation of the hydrophilic methylene blue into a liposome core. Taken from ref. 156. Published by MDPI; (C) previous encapsulation of a hydrophobic cargo into cyclodextrins, and incorporation into a liposome. Taken from ref. 157. Reproduced with permission from the National Academic of Sciences, Copyright $\mathbb{C} 2014$.

effects, and helped load the porphyrin into the liposome lipid bilayer. ${ }^{153}$ Similar strategies have been used to encapsulate BODIPY-based PSs in lipidic structures. ${ }^{154}$ However, the PSs can also be incorporated after incubation with already formed liposomes, as it happens with the hydrophilic 5-aminolevulinic acid (ALA), ${ }^{155}$ and also with methylene blue (Fig. 5B). ${ }^{156}$ This second strategy is known as active loading. In some cases, highly hydrophobic and nonionizable cargos can be previously encapsulated into cyclodextrins, and later be incorporated into the liposomes (Fig. 5C). ${ }^{157,158}$

Liposomes are well-known drug carriers, and in fact, some PSs are commercially available as liposomal formulations. Visudyne ${ }^{\circledR}$ was one of the first nanoformulations of photosensitizers. It consists of a liposomal formulation of verteporfin, a semisynthetic porphyrin derived from protoporphyrin, and it was approved by the FDA in 2000 for the treatment of age-related macular degeneration (AMD), and in 2001, for the treatment of pathological myopia. ${ }^{159}$ Foslip $^{\circledR}$ is a more recently developed third-generation PS based on a liposomal formulation of 
m-THPC, a chlorine-derivative PS. This formulation reduced damage of healthy tissues, compared to the m-THPC nonliposomal formulation Foscan ${ }^{\circledR}$, in which the drug is dissolved in a mixture of water-free ethanol and propylene glycol. ${ }^{160}$ M-THPC has also been encapsulated into a PEGylated lipid. This formulation is commercially known as Fospeg ${ }^{(\mathbb{B})}$ and offers additional stability and a longer circulatory half-life. ${ }^{161}$ In general terms, the delivery of m-THPC by liposomes significantly abolished its dark cytotoxicity, slightly improved the cellular uptake and, following PDT, promoted cell loss and spheroid disassembling to a higher extent when compared to that of Foscan ${ }^{\circledR}{ }^{162}$ The encapsulation of $\mathrm{m}$-THPC into extracellular vesicles has been correlated with higher stabilities, and accumulation and penetration in multicellular tumour spheroids. ${ }^{163}$ A commercial liposome-based formulation of 5-aminolevulinic acid (5-ALA) (PhotoSpray, Ellipse ${ }^{\circledR}$ ) is also used for skin treatments of photorejuvenation and wrinkle reduction. $^{164}$

Liposomes can be rationally designed for controlled cargo release, for the decrease of their clearance, and also to direct them to the target site in the human body. Such features are connected with the attenuation of the possible side effects of the PDT treatment, when compared to conventional liposomes. ${ }^{165}$ For example, the use of functionalised lipids or other functional structures that provide additional stabilization can increase the circulatory life of the liposomes, and thus overcome one of the possible limitations of such systems. In this way, these carriers can better accumulate the therapeutically cytotoxic concentration of the drug within the tumour tissue. This is in fact one of the features of the commercial liposome formulation Fospeg ${ }^{\circledR}$, which belongs to the long-circulating or "stealth" liposome category. The incorporation of $\mathrm{pH}$-sensitive molecules that change their charge according to the $\mathrm{pH}$ of the medium is used to design $\mathrm{pH}$-sensitive liposomes. Such formulations are designed for a better release of the cargo. Other strategies such as the use of cationic liposomes, which in some cases has been shown to exhibit similar PDT efficiency but less tissue damage, ${ }^{166}$ and the incorporation of antibodies for active targeting (immunoliposomes) have also been reported.

The incorporation of high concentrations of cargos is currently one of the challenges in the chemistry of liposomes. One possible improvement in this direction has been reported for liposomes loaded with tetraphenylporphyrin (TPP) that can be incorporated into a liposome at high concentrations via an exchange reaction from previously formed cyclodextrin/ porphyrin complexes. ${ }^{158}$

With the objective of multifunctionality, some PSs such as the hydrophobic pyropheophorbide acid, a chlorophyll derivative which can be used either as a PS or as a fluorescent reagent, are encapsulated into a liposome structure and can be employed for imaging-guided photodynamic therapy. ${ }^{167}$ As previously commented, another advantage of liposomes is the possible incorporation of both hydrophilic and hydrophobic cargos, and this allows the design of multi therapy systems. This is, for example, explored in the incorporation of the hydrophilic metformin, a compound that improves tumour oxygenation, into chlorin e6 (HCe6)-loaded liposomes. In this case, several phospholipids, which can be functionalised with PEG in the presence of HCe6 (eventually located inside the formed lipid bilayer), are mixed and dried and are eventually incubated with an aqueous solution of metformin. ${ }^{168}$ A similar strategy was used to co-encapsulate a chlorin derivative and AQ4N, a hydrophilic hypoxia-activated prodrug that improves the cancer treatment with hypoxia-activated chemotherapy, given that tumour hypoxia is a characteristic feature of most solid tumours. $^{169}$

As in many other cases, within drug delivery, a controlled release of the cargo is one of the research topics that attracts wide attention. This is, for example, considered in the encapsulation of aggregation-induced emission PSs, that allows controlled photosensitization. Such PSs are hydrophobic and can be entrapped into the liposomal lipid bilayer, where they lose their photosensitivity. Once the liposomes have reached the tumour tissue and decompose, the released PSs are able to reaggregate and recover their properties. ${ }^{170}$

\section{3c. Metal-organic frameworks}

Metal-organic frameworks (MOFs) are assembled from metal ions (or clusters) and organic linkers. These structures have been gaining increasing interest in the last few years for a myriad of bioapplications, ${ }^{171,172}$ mainly by virtue of their high specific surface area, and exceptional tunability in terms of structural and chemical composition by varying the metal nodes and organic linkers. ${ }^{173-175}$ Such a high degree of flexibility in structural design allows adjustment of the pore size, hydrophobic/hydrophilic nature of pores, flexibility of the structure to accommodate molecules with larger sizes than their aperture pore size, and post-synthetic modifications for incorporating additional functionalities. ${ }^{176,177}$ Within the biomedical field, the potential of MOFs as ideal nanocarriers has been extensively investigated, demonstrating their broad applicability to very diverse cargo molecules such as drugs, gases, imaging probes, as well as PSs. ${ }^{171,178-181}$

In the particular case of PDT, the main advantages of MOFs can be summarized as follows: ${ }^{180,181}$ (i) their exceptional structural and compositional tunability allows the incorporation of a myriad of PSs with a high PS loading capacity thanks to their remarkable high surface area; (ii) their structural regularity (i.e., well-defined porosity) allows confined PSs to isolate from each other, which is quite important for avoiding self-quenching that would diminish their therapeutic efficiency; (iii) rather than encapsulating the PS molecules into the pores, photosensitizing organic ligands (e.g., porphyrins ${ }^{182}$ and BODIPY $^{183,184}$ ) can also be incorporated as linkers within the MOF structure; (iv) their high porosity facilitates the diffusion of ROSs, allowing ${ }^{3} \mathrm{O}_{2}$ and ${ }^{1} \mathrm{O}_{2}$ to diffuse freely in and out of the framework; (v) their intrinsic biodegradability ${ }^{185}$ and minimal toxicity ${ }^{171,179}$ by judicious choice of their composition (i.e. metal ions and linkers), as well as the possibility to be synthesized at the nanoscale (i.e., nanoscale MOFs (nMOFs)), ${ }^{171,178,186}$ provide MOFs with key requirements for bioapplications; and (vi) nMOFs can be designed to be degraded/decomposed upon endogenous 
chemical triggers (e.g., $\left.\mathrm{pH}, \mathrm{GSH}^{187,188}\right)$ or even to be activated by external energy stimuli (i.e. light ${ }^{189}$ ), which is highly desirable for the development of activable PS nanoplatforms.

While the above-mentioned nanoparticle-based PSs have met limited success in PDT due to the difficulty in simultaneously optimizing ROS generation and targeted delivery to intracellular organelles to exert cytotoxic effects, nMOFs can be rationally designed to play multiple synergistic functions/roles without relying on cytotoxic agents, which often results in severe general toxicity. Therefore, the multifunctionality potential of nMOFs is the strong distinctive point to make the difference. By combining the advantageous features of MOFs, a plethora of PS-based MOF nanoplatforms for PDT have been designed, and many of them yielded quite promising results as it will be illustrated in the following by means of some selected examples.

In 2014, Lin and co-workers reported for the first time the use of porphyrin-based MOFs to address some of the limitations of PDT in difficult-to-treat cancers, such as the low accumulation of PSs in tumours and the low PDT efficiency because of PS aggregation and limited ${ }^{1} \mathrm{O}_{2}$ diffusion. ${ }^{190}$ They prepared Hf-porphyrin nMOFs, consisting of Hf-oxo clusters and 5,15$\operatorname{di}\left(p\right.$-benzoato)porphyrin $\left(\mathrm{H}_{2} \mathrm{DBP}\right)$ as ligands, which efficiently generated ${ }^{1} \mathrm{O}_{2}$ due to site isolation of porphyrin ligands, enhanced intersystem crossing by heavy $\mathrm{Hf}$ centers, and facilitated ${ }^{1} \mathrm{O}_{2}$ diffusion through the porous structure. Consequently, these nMOFs presented a good PDT efficacy both in vitro and in vivo by using murine head and neck squamous cell SQ20B, leading to complete tumour eradication in half of the mice receiving a single nMOF dose and a single light exposure. Although this approach of incorporating the PS as a linker within the MOF framework is only applicable to a limited number of PSs, whichever PS can, in principle, be encapsulated during the formation of the MOF or further introduced by following post-synthetic modifications. In this way, different kinds of PSs (e.g., Ce6, TPEDC, and TPETCF) were successfully encapsulated into MIL-100(Fe) and proposed as a general strategy to yield activatable PSs. ${ }^{191}$ While the PSs were encapsulated into the MIL-100, their photosensitization capability was suppressed due to their isolation from $\mathrm{O}_{2}$. Upon reaction between iron(III) in MIL-100 and intracellular $\mathrm{H}_{2} \mathrm{O}_{2}$ (Fenton reaction), the framework of $\mathrm{MIL}-100(\mathrm{Fe})$ collapsed, releasing the PSs, and thereby activating photosensitivity by regaining contact with $\mathrm{O}_{2}$. Particularly, the TPETCF@MIL-100 system was tested both in vitro and in vivo on $4 \mathrm{~T} 1$ cells and $4 \mathrm{~T} 1$ tumour-bearing mouse models, which displayed an enhanced PDT efficiency compared to that of the free TPETCF upon white light irradiation. Similarly, phthalocyanine PS ( $\mathrm{ZnPc}$ ) was encapsulated into the micropores of ZIF-8. ${ }^{192}$ Under weak acidic conditions ( $\mathrm{pH}$ 5.0) found in tumour microenvironments, ZIF-8 was decomposed to release $\mathrm{ZnPc}$, which induced the production of ${ }^{1} \mathrm{O}_{2}$ in cells after $650 \mathrm{~nm}$ light irradiation, resulting in the PDT effect. Despite the fact that these last examples seem effective, since the nMOF pores are filled with the PS, the potential use of these nanosystems for combinatorial therapy, for example, by introducing chemotherapeutics, is compromised. In this sense, and with the goal of developing multifunctional nanoplatforms that integrate different therapeutic modalities, such as chemotherapy and PDT, in one single nanoparticle to pursue high therapy effectiveness, the integration of the PS as a building unit of the nMOF is a more beneficial approach.

Although the examples discussed so far led to improved PDT effects, the therapeutic efficiency of PDT in a real scenario is severely limited by the low concentration of $\mathrm{O}_{2}$ in solid tumours (i.e. tumour hypoxia), which occurs because the oxygen supply is reduced by the uncontrolled proliferation of cancer cells. ${ }^{193,194}$ Moreover, the vascular damage produced during the PDT process further worsens hypoxia, and in turn limits the PDT efficiency. ${ }^{195}$ As a result, tumour hypoxia is known as the "Achilles' heel" of traditional PDT. ${ }^{196}$ Various nMOFs relying on different strategies have been explored to address the limitation of tumour hypoxia, which can be grouped into two types: oxygen self-supplement systems to promote in situ oxygen generation, and "oxygen shuttles" to carry and deliver oxygen into tumours. As an example of the first strategy, Fe-based MOFs able to decompose intracellular hydrogen peroxide $\left(\mathrm{H}_{2} \mathrm{O}_{2}\right)$ for the in situ generation of $\mathrm{O}_{2}$ were reported. ${ }^{197} \mathrm{Fe}-\mathrm{TBP}$ MOFs, assembled from $\mathrm{Fe}_{3} \mathrm{O}$ clusters and 5,10,15,20-tetra( $p$-benzoato) porphyrin (TBP) ligands, catalysed a cascade reaction when irradiated under hypoxic conditions, in which intracellular $\mathrm{H}_{2} \mathrm{O}_{2}$ was decomposed by the $\mathrm{Fe}_{3} \mathrm{O}$ clusters to produce $\mathrm{O}_{2}$ through a Fenton-like reaction, whereas the generated $\mathrm{O}_{2}$ was converted to cytotoxic singlet oxygen $\left({ }^{1} \mathrm{O}_{2}\right)$ by photoexcited porphyrins. After PDT treatment, Fe-TBP effectively regressed locally irradiated tumours of hypoxic CT26 colorectal adenocarcinoma. Another reported approach for generating $\mathrm{O}_{2}$ in situ inside the tumour is based on the immobilization of platinum nanozymes (i.e. Pt NPs with catalase-like activity) on photosensitizer-integrated MOFs, particularly porphyrinic ZrMOF nanoparticles (PCN-224). ${ }^{198}$ In vivo PDT via intratumoural and intravenous injection of these $\mathrm{PCN}-224-\mathrm{Pt}$ nanocomposites showed enhanced PDT efficiency via $\mathrm{H}_{2} \mathrm{O}_{2}$-activated evolvement of $\mathrm{O}_{2}$ and light-irradiated formation of ${ }^{1} \mathrm{O}_{2}$ (Fig. 6A) at the tumour site. In this regard, ZIFs (a subfamily of MOFs) and $\mathrm{Cu}$-doped ZIFs can serve as an $\mathrm{O}_{2}$ reservoir to quickly release $\mathrm{O}_{2}$ once reaching the tumour by exploiting their $\mathrm{pH}$-triggered decomposition at the acidic tumour microenvironment (Fig. 6B). ${ }^{199,200}$

Unfortunately, due to the severe hypoxia existing in malignant tumours, $\mathrm{O}_{2}$ supply is still less effective for type II PDT. Different from the type II route, some recent approaches are focused on the development of nMOFs with a potential to promote type I PDT, which consists of the directly activated reactions between PS and substrates via a hydrogen- or electron-transfer process and usually produces hydroxyl radicals $\left({ }^{\bullet} \mathrm{OH}\right)$ or superoxide radicals $\left(\mathrm{O}_{2}{ }^{\bullet-}\right)$. For example, Lin and co-workers recently designed a Ti-TBP nMOF, composed of Ti-oxo chain secondary building units and photosensitizing porphyrin ligands (particularly 5,10,15,20tetra( $p$-benzoato)porphyrin (TBP)), which enabled hypoxiatolerant type I PDT (Fig. 7). ${ }^{201}$ Upon light irradiation, in addition to sensitizing ${ }^{1} \mathrm{O}_{2}$ production, Ti-TBP generated superoxide $\left(\mathrm{O}^{2-}\right)$, hydrogen peroxide $\left(\mathrm{H}_{2} \mathrm{O}_{2}\right)$, and hydroxyl radicals $\left({ }^{\bullet} \mathrm{OH}\right)$ via transferring electrons from excited $\mathrm{TBP}^{*}$ species to $\mathrm{Ti}^{4+}$ units to form $\mathrm{TBP}^{\bullet+}$ ligands and $\mathrm{T}^{\mathrm{i} 3+}$ centers. It is worth noting that such an 


\section{(A) in situ $0_{2}$ generation}

a1

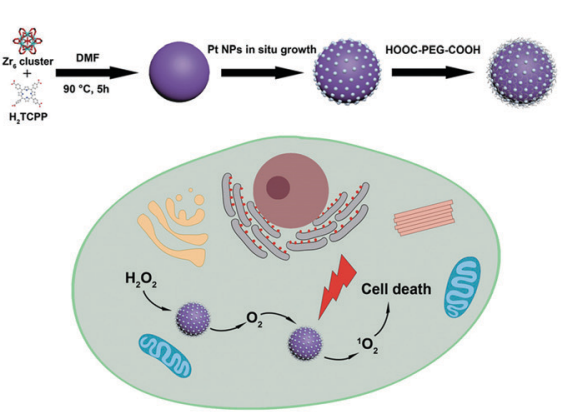

a2

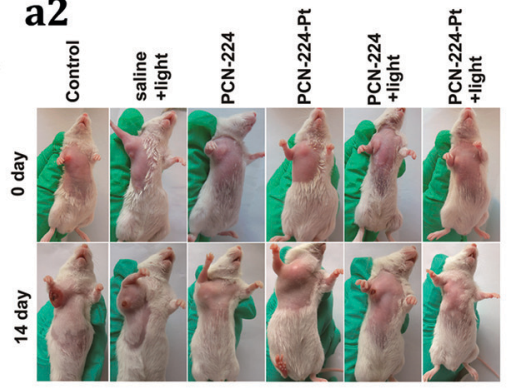

a3

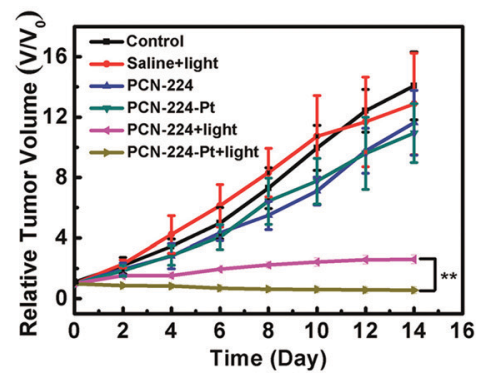

(B) $\mathrm{O}_{2}$-loaded nanoplatform

b1

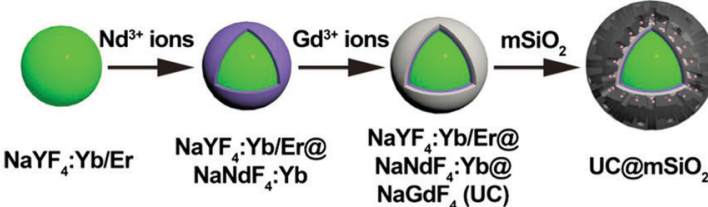
$\mathrm{NaGdF}_{4}$ (UC)
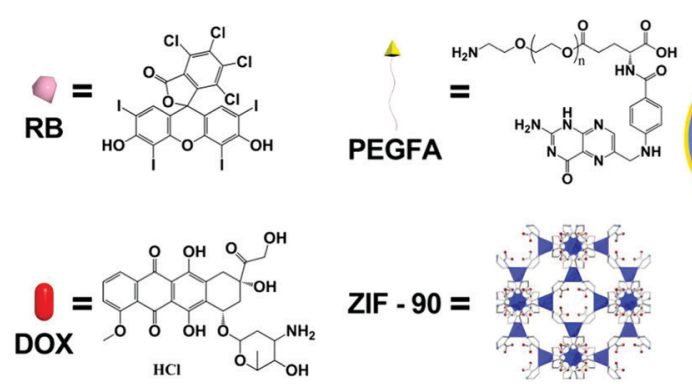

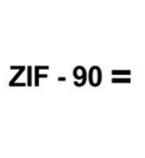

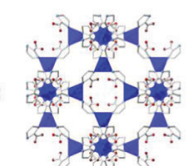

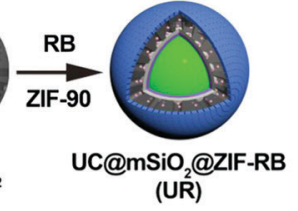

$\mathrm{O}_{2} \underset{\mathrm{PEGFA}}{\mathrm{DOX}}$

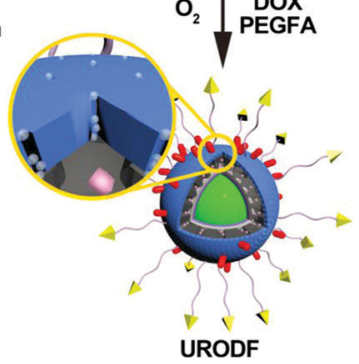

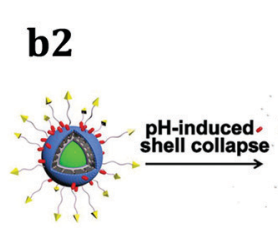

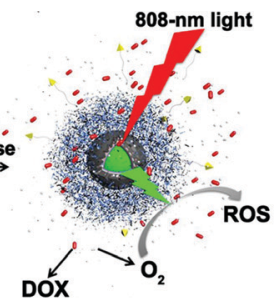

b3

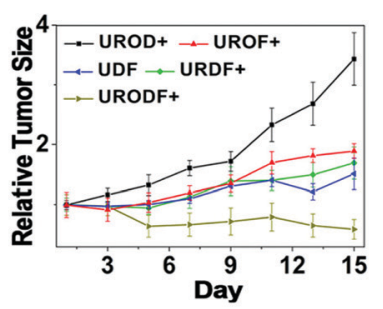

Fig. 6 Two examples of MOF-based approaches for overcoming tumour hypoxia in PDT type II. (A) In situ $\mathrm{O}_{2}$ generation at the tumour: (a1) scheme of the preparation of porphyrinic Zr-MOF with immobilised Pt NPs as nanoenzymes (PCN-224-Pt); (a2) schematic illustration of PCN-224-Pt performance for enhanced PDT; (a3) photographs of the $\mathrm{H} 22$ tumour-bearing mice before treatment and on day 14 after various treatments; and (a4) relative tumour volume after various treatments. Adapted from ref. 198 with permission from the American Chemical Society, Copyright $\left(\mathbb{C} 2017 .(B) \mathrm{O}_{2}-\right.$ loaded nanoplatform to carry and deliver $\mathrm{O}_{2}$ at the tumour: (b1) schematic illustration of the preparation of UC(amSiO 2 -RB@ZIF-O2-DOX/PEGFA (URODF) nanoparticles; (b2) scheme of URODF nanoparticle performance as a $\mathrm{pH}$-sensitive double-drug-loaded nanodrug carrier for $\mathrm{O}_{2}$-enhanced PDT and chemotherapy; (b3) tumour volume growth curves; and (d) dynamic body change of mice in UROD+, UROF+, UDF, URDF+, and URODF+ treated groups during a period of 14 days. Adapted from ref. 200 with permission from the American Chemical Society, Copyright $\mathbb{C} 2018$.

electron transfer (ET) process was facilitated by the proximity of Ti-oxo chains to TBP ligands $(\sim 1.1 \mathrm{~nm})$. As a result, four distinct ROSs were generated, thus leading to outstanding anticancer efficacy with $>98 \%$ tumour regression and $60 \%$ cure rate.

Another key issue that limits the PDT efficiency is the overexpression of glutathione (GSH) in cancer cells, which significantly reduces the cytotoxicity of ROS during the treatment. A straightforward strategy to solve this involves using nanomaterials for intracellular oxidation reactions to reduce intracellular GSH levels. Following this idea, Tang and co-workers reported for the first time a $\mathrm{Cu}(\mathrm{II})$-based porphyrinic MOF where the $\mathrm{Cu}^{2+}$ were able to adsorb intracellular GSH. ${ }^{201}$ Interestingly, those $\mathrm{Cu}^{2+}$ active centers had a dual function: reducing the GSH levels, and simultaneously serving as a PDT photosensitizer, resulting in increased levels of ROS and consequently enhancing the effects of PDT. More recently, Lin and co-workers developed a copper-based MOF as a PDT nanosystem for overcoming hypoxia and reducing intracellular GSH at the same time. ${ }^{202}$ They designed a biocompatible and biodegradable $\mathrm{O}_{2}$-loaded CuTz-1@F127 MOF that could act as a light-activated photosensitizer (PS) to generate hydroxyl radicals $\left({ }^{\bullet} \mathrm{OH}\right)$ and $\mathrm{O}_{2}$ in the presence of hydrogen peroxide $\left(\mathrm{H}_{2} \mathrm{O}_{2}\right)$, i.e., type I PDT, under NIR irradiation. In addition, MOF nanoparticles could release adsorbed $\mathrm{O}_{2}$ to alleviate intracellular hypoxia, and also react with intracellular GSH by means of the $\mathrm{Cu}^{+}$within the framework. In vitro and in vivo studies demonstrated the high efficacy of PDT of cancers by using this MOF-based system. Importantly, the coating with the amphiphilic polymer F127 enhanced the biocompatibility of the MOF, as confirmed by in vivo long-term toxicity, biodistribution and excretion experiments.

Light penetration is another major obstacle to improving the efficacy of PDT, and makes PDT unsuitable for deep-seated tumours. To solve this issue, several research groups have made significant contributions to the development of nMOFs 
a

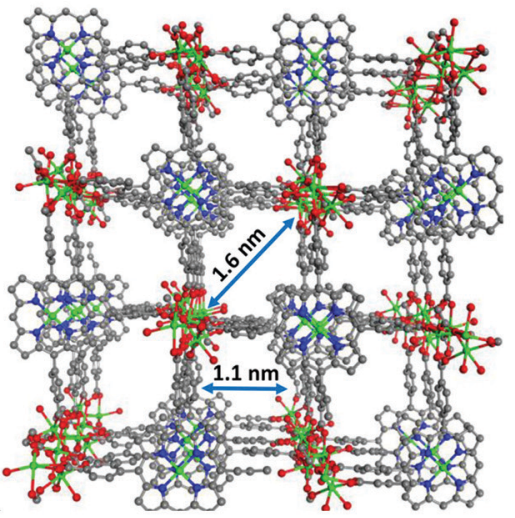

b $\mathrm{O}_{2}$

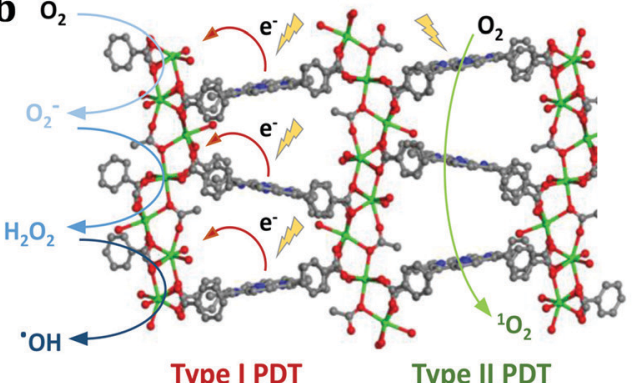

c
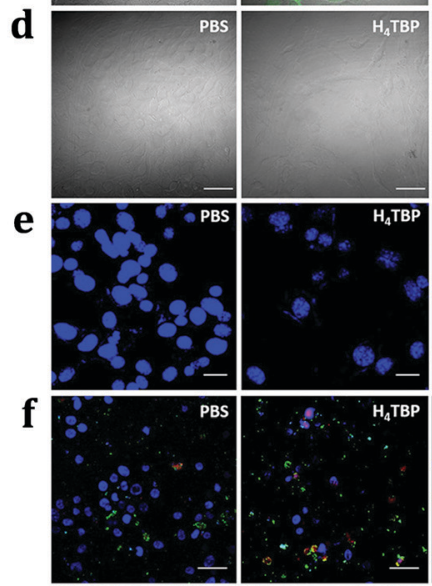

g

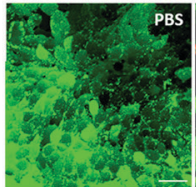

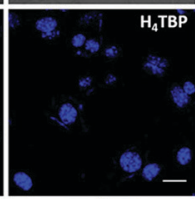
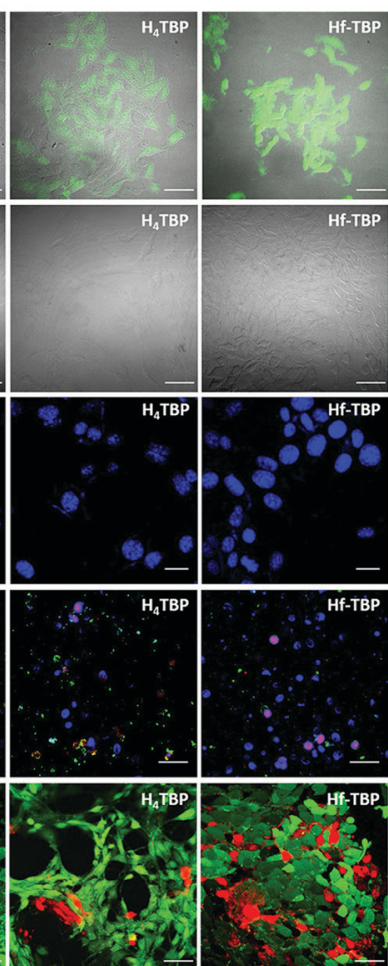

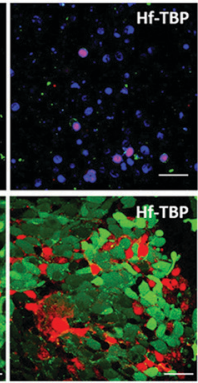

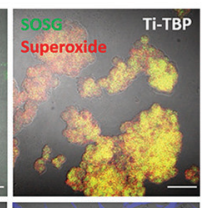
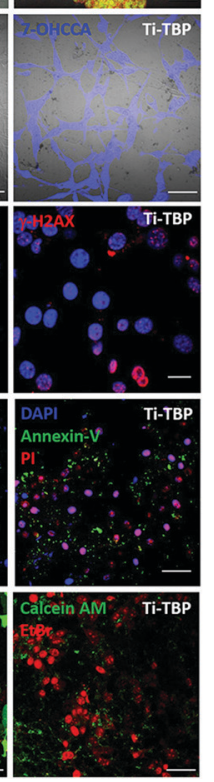

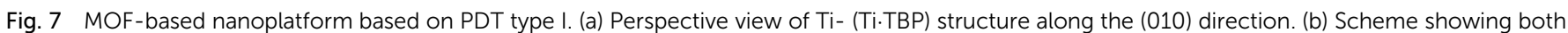

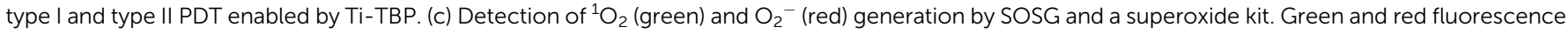

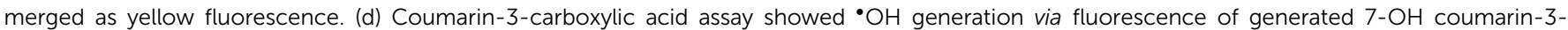

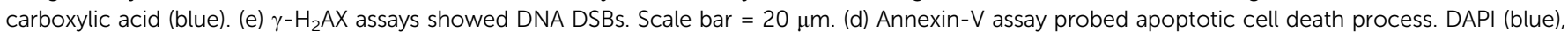

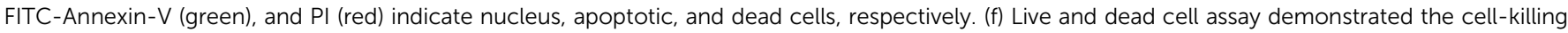

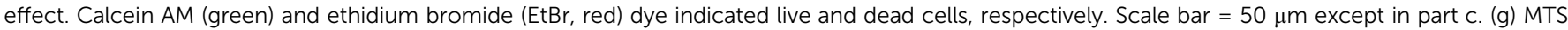

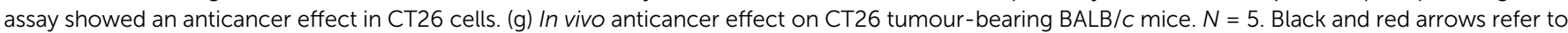

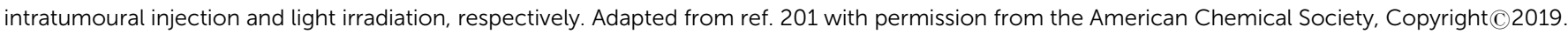

containing lanthanide nanoparticles to realize near-infrared (NIR)-excited PDT via an upconversion process. One interesting example reported the synthesis of heterodimers through the selective anisotropic growth of Zr-based porphyrinic nMOFs onto UCNPs (UCMOFs). ${ }^{203}$ Such asymmetric nanostructures could harvest photons beyond the absorption spectra of the native porphyrinic MOFs via the resonance energy transfer from the UCNPs to nMOFs, resulting in the production of ${ }^{1} \mathrm{O}_{2}$ under NIR light irradiation. Furthermore, by loading the anticancer drug doxorubicin (DOX) into the porous channels of the nMOF, this nanoplatform enabled the combination of chemotherapy and NIR-induced PDT. In vivo studies indicated that the tumour growth of mice treated with UCMOFs + NIR was efficiently delayed due to the PDT effect, while in the DOX/UCMOFs + NIR treated group, the tumour growth was completely suppressed, which clearly pointed out that the combination of chemotherapy and PDT is more effective than either modality alone. Similarly, an $\mathrm{O}_{2}$-loaded $\mathrm{pH}$-responsive multifunctional nanodrug carrier UCNPs@mSiO ${ }_{2}$-RB@ZIF-O ${ }_{2}$-DOX-PEGFA (URODF) with enhanced chemo-photodynamic therapeutic effect was fabricated (Fig. 6B). ${ }^{200} \mathrm{NaYF}_{4}: \mathrm{Yb} / \mathrm{Er} @ \mathrm{NaYbF}_{4}: \mathrm{Nd} @ \mathrm{NaGdF}_{4}$ UCNPs were employed for dual-modal upconversion/magnetic resonance (MR) imaging, whereas the mesoporous silica $\left(\mathrm{mSiO}_{2}\right)$ shell served to encapsulate the photosensitizer Rose Bengal (RB).
This core-shell structure allowed UCNPs to harvest $808 \mathrm{~nm}$ photons and achieve green emission through the multiphoton process, thereby activating the PS in the therapeutic window. Under acidic conditions, the outmost $\mathrm{O}_{2}$ reservoir ZIF-90 shell could decompose, allowing quick release of $\mathrm{O}_{2}$ and DOX at low pH in the tumour microenvironment, and therefore achieving improved synergetic therapy and alleviating tumour hypoxia. In vitro cytotoxicity against $4 \mathrm{~T} 1$ and HeLa cells, and in vivo tumour inhibition studies against $\mathrm{H} 22$ cancer cells, demonstrated a remarkably enhanced tumour inhibition effect by URODF NPs.

Despite the progress made in the field of nMOFs for PDT, toxicity remains a major concern before nMOFs can be tested on humans. However, the fact that a nMOF formulated for radio-enhancement is already under clinical trial (NCT 03444714) ${ }^{204}$ may set the stage for other promising nMOFs to be carefully tested for their therapeutic efficacy with the ultimate goal of entering clinical trials. In conclusion, even if many more efforts are still needed to unlock the full potential of nMOFs in PDT, on the one hand, and to solve the toxicity issues, on the other hand, the future of nMOFs in PDT as well as in other cancer therapies is bright.

\section{3d. Targeting moieties}

As in many other nanostructured systems, the incorporation of PSs paves the way for additional and improved features of the 
resulting material, offering the possibility of multifunctionality and active targeting, among other features. ${ }^{19}$

Nanostructures themselves take advantage of the enhanced permeability and retention effect (EPR), and tend to accumulate in tumour and damaged tissues. Such effect is known as passive targeting, which usually helps direct nanostructures to tumour cells. However, in most of the cases, this gives rise to drug concentrations that are not sufficient for the therapy of most cancers. ${ }^{205}$ The active targeting consists of the incorporation of specific molecules or species, named targeting moieties, which enhance the internalization of the nanostructures with certain types of cells. This effect is based on the interactions of the moieties with receptors overexpressed on the cell surface, especially on tumour cells. Such moieties are specific for certain types of tumour cells, and are mostly based on antibodies, carbohydrates, lectins, peptides, folic acid and different aptarmers. $^{206-208}$ Carbohydrates are involved in many cell-cell recognition processes. ${ }^{209-211}$ For example, mannose-6phosphate has been used to target prostate tumour cells. ${ }^{212}$ Some sugar such as galactose and lactose can also be used as targeting ligands for hepatic tumour cells, ${ }^{213-215}$ while folic acid is used to target ovarian cancer. ${ }^{216}$ Other biomoleculerelated targeting moeities can also be found in the literature, as in the case, for example, of peptides for the targeting of prostate cancer. $^{217}$ Different targeting moieties have been incorporated into nanostructures designed to be used in PDT, and some of the recent examples that can be found in the literature will be discussed in this section.
A porphyrinic metal-organic framework, in which NPs are functionalised with folic acid, has been designed to target ovarian tumours. In this nanostructure, the folic acid was conjugated via its carboxylate end with the MOF cluster, which possesses available binding sites (Fig. 8a). ${ }^{182}$ Sugar-based moieties such as galactose derivatives, showing a great affinity for receptors overexpressed in liver cancer cells, ${ }^{215}$ can be incorporated into nanostructures. The general strategy here consists of the prior incorporation of the sugar moieties into the block copolymers forming the micelle, prior to selfassembly. ${ }^{139,218}$ Integrin $\alpha_{\mathrm{v}} \beta_{3}$ monoclonal antibodies can be incorporated into nano graphene oxide via EDC chemistry, and are used to target $\alpha_{v} \beta_{3}$ positive tumour cells. In this system, the PS is the pyropheophorbide-a, which is conjugated with PEG, and is later used to cover the graphene oxide surface. ${ }^{219}$ Similar strategies were reported for a porphyrin derivative-PEG-coated nanographene oxide functionalized with folic acid. ${ }^{220}$

Targeting peptides can also be found in the literature. For example, gold nanoparticles functionalised with a thiol-ending PEG conjugated with an epidermal growth factor peptide have been synthesised for the therapy of brain cancer. In this case, a hydrophobic phthalocyanine-based PS is non-covalently adsorbed onto the Au NP surface with the help of PEG (Fig. 8b). ${ }^{221}$

The coating of UCNPs with $\mathrm{SiO}_{2}$ also opens up the way to many subsequent functionalization possibilities. For example, the PS Rose Bengal can be incorporated into the silica shell, and the bioconjugation of a monoclonal antibody for epithelial
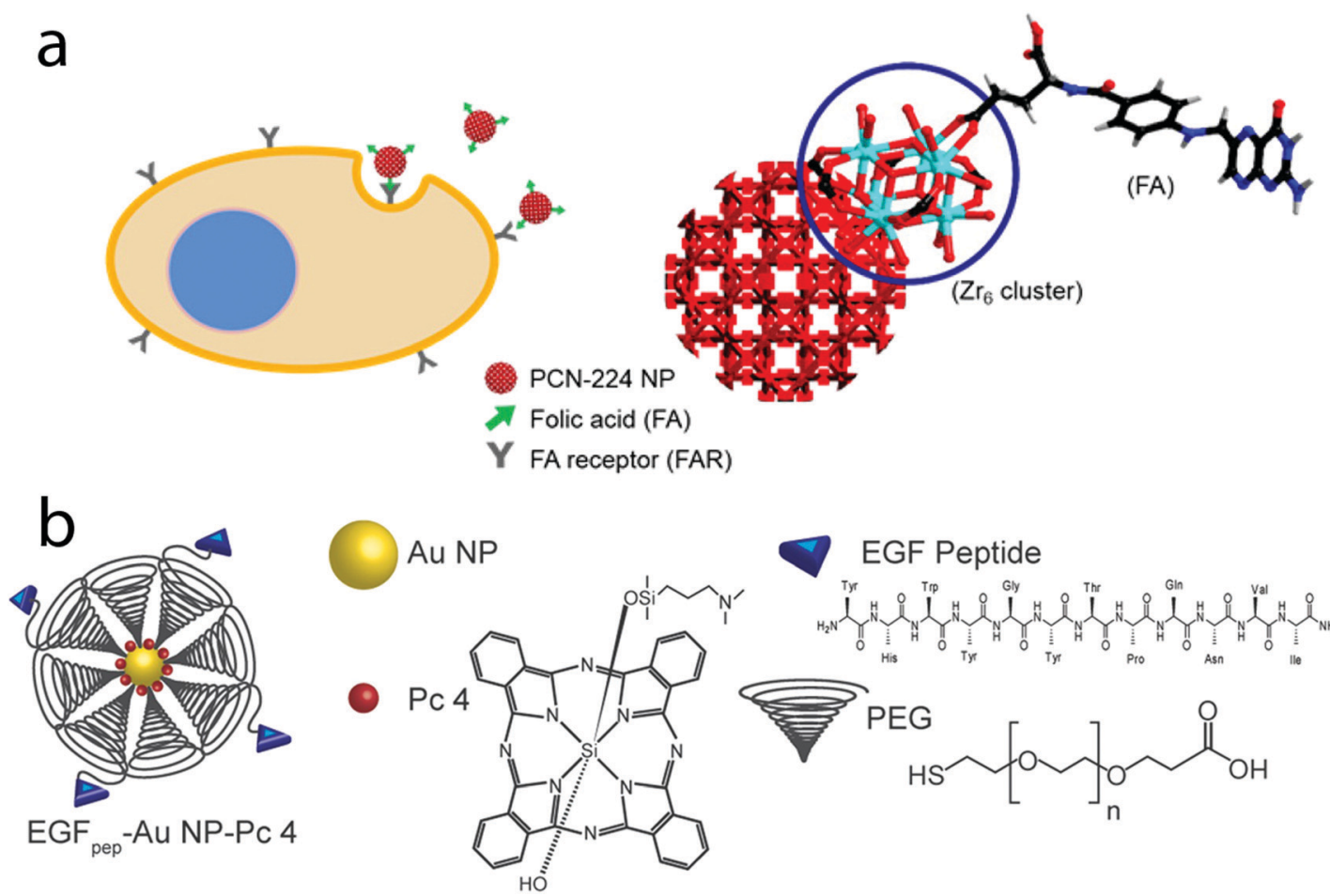

$\nabla$ EGF Peptide
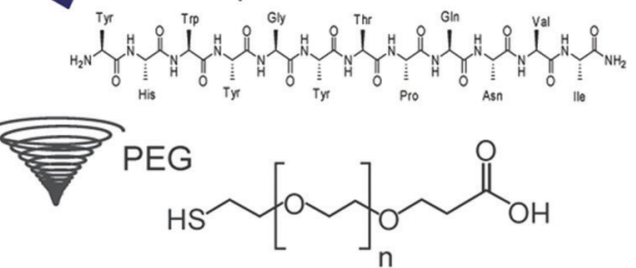

Fig. 8 (a) MOF NPs containing a Zr porphyrin and functionalised with folic acid (FA). Taken from ref. 182. Reproduced with permission from the American Chemical Society, Copyright (C)2016; (b) Au NPs functionalised with a targeting peptide (EFG)-ending PEG, and containing the phthalocyaninebased PS Pc 4. Taken from ref. 221. Reproduced with permission from John Wiley \& Sons, Copyright $(2015$. 
cell adhesion molecules (EpCAM, also known as CD326) on a silica-specific solid-binding peptide facilitates the incorporation of tumour-targeting antibodies onto the silica-coated surfaces. This is used to target and treat human colorectal adenocarcinoma HT-29 cells. As in the previous example, such UCNPs allow the production of ROS by excitation with NIR radiation, and also show luminescence properties. ${ }^{22}$

Not only tumour cells but also cellular organelles can be targeted. The special role played by the mitochondria ${ }^{223}$ has recently paved the way for the development of new cancer therapies. In the same direction, the cellular nucleus is one of the most common biological targets for many anticancer therapies. ${ }^{224}$ In this context, it is known that some peptides are used as vectors for the mitochondria targeting delivery in cancer stem cells, in which its mitochondrial activity plays a key role, and its regulation has thus been suggested as a new therapeutic strategy to activate cell death. ${ }^{225}$ Based on this, the conjugation of both the protoporphyrin IX and PEG with an amphiphilic mitochondria-penetrating peptide, forming micelles after self-assembly, is employed for the PDT in mitochondria. ${ }^{226}$ Analogously, the conjugation of an $\operatorname{Ir}(\mathrm{III})$ PS complex with human serum albumin (HSA) nanoparticles via its histidine and cysteine residues has been reported to target the nucleus of living cancer cells. ${ }^{227}$

\section{Perspectives and possible evolutions}

Despite the considerable progress made over the last ten years in this field, and the significant advantages of PDT to treat certain types of cancers and pre-cancers compared to conventional chemotherapy and radiation therapy, PDT has not become a mainstream cancer therapy. This is due to a number of drawbacks that have to be overcome before the translation of these PDT-based nanostructures into clinical practice in the future. These issues are mainly the following: (i) PDT is not suitable to treat large or deep-seated tumors since light cannot penetrate deeply through tissues. In this sense, NIR light penetrates deeper into tissues than UV and visible light, and thus, PSs excited with NIR light are desirable; (ii) the fact that PDT is a local treatment makes it not useful for treating metastatic cancers; and (iii) patients treated with PDT typically report high sensitivity to light over a period of time, requiring special precautions after PDT treatment. Current efforts to address these limitations can be grouped mainly into one of the following two approaches: the search for more effective PSs, or the development of new or improved nanostructures capable of improving the localization of PSs in tumors.

Within the first approach, intensive research is devoted to improving the properties of PSs, either by synthesizing new compounds or through modifications of the core of already existing ones, with the aim of obtaining PSs with higher absorption in the optical window, larger extinction coefficients, more efficient singlet oxygen production, excitable with NIR light, and better chemical and physical properties, especially appropriate solubility. It should also be considered that the key property related to the nanostructured system for controlling the location of the PS is its targeting ability. Carbohydrates, proteins, peptides, and antibodies are the most common targeting molecules attached to the surface of the nanostructured carrier system to realize active targeting of PSs.

Multifunctionality is another promising evolution of such systems, which already in some cases, and depending on the complexity and components of the nanostructure, can be used for different therapies (chemotherapy, PDT, and PTT), and also as luminescent probes. Examples of this are micelles loaded with both PSs and drugs, aiming to overcome drug resistance (Fig. 9). ${ }^{228}$

Other multifunctional nanostructures can already be found in the recent literature. For example, the functionalization of lanthanide-based UCNPs with a hydrophobic polymer and the hydrophilic PEG creates a micelle into which the hydrophobic PS Rose Bengal, as well as other hydrophobic drugs, can be incorporated. In this case, a pansomatostatin synthetic

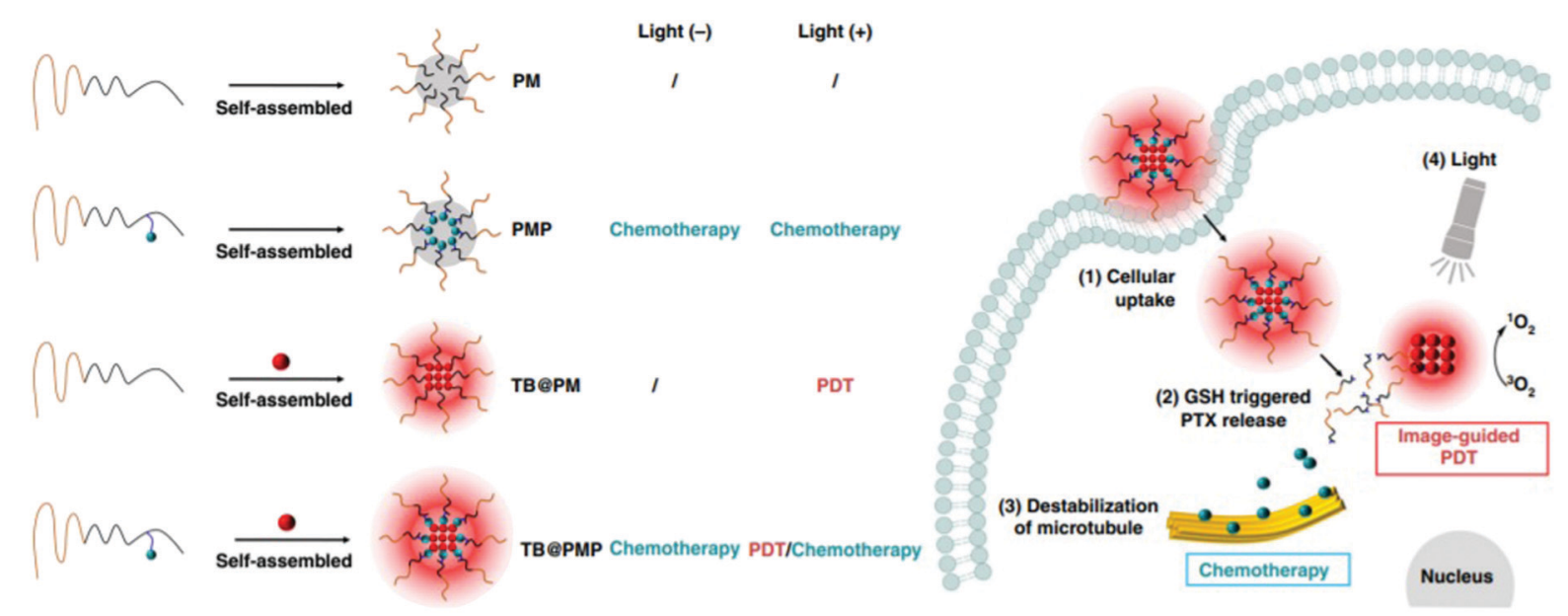

Fig. 9 Micelles designed for both PDT and chemotherapy, depending on the structure and loading. Taken from ref. 228. Published by Springer Nature, 2018. 
nonapeptide is conjugated with PEG, which serves as a tumourtargeting ligand for medullary thyroid cancers. Such nanostructures combine chemotherapy, NIR-activated PDT and fluorescence imaging, and also allow controlled drug release, based on a hydrophobic to hydrophilic transition in the polymer shell under irradiation (Fig. 10). ${ }^{229}$

Another interesting example of multimodal therapeutic nanoplatforms based on nMOFs has been discussed above (Fig. 6B), ${ }^{200}$ where chemotherapy and PDT are combined. Similar nanoplatforms have also been designed for the combination of PDT and chemodynamic therapy (CDT), which is an emerging nanocatalyst-based therapeutic modality based on Fenton or Fenton-like reactions. For example, Lin and co-workers have recently constructed a core-shell nanostructure, $\mathrm{O}_{2}-\mathrm{Cu} / \mathrm{ZIF}$ 8@Ce6/ZIF-8@F127 (OCZCF), to enhance the PDT and CDT efficacy by relieving hypoxia and consuming GSH. ${ }^{199}$ ZIF-8 can be decomposed in the acidic tumour microenvironment and release $\mathrm{O}_{2}$, Ce6, and $\mathrm{Cu}^{2+}$ simultaneously. The released $\mathrm{O}_{2}$ could be converted into ${ }^{1} \mathrm{O}_{2}$ by $\mathrm{Ce} 6$ under irradiation of a $650 \mathrm{~nm}$ laser. Moreover, the released $\mathrm{Cu}^{2+}$ can act as a smart reactive oxygen species protector by consuming intracellular GSH via oxidation, and thereby producing GSSG and $\mathrm{Cu}^{+}$. The byproduct $\mathrm{Cu}^{+}$can further catalyse the decomposition of $\mathrm{H}_{2} \mathrm{O}_{2}$ to generate ${ }^{\bullet} \mathrm{OH}$ Fenton-like reactions for enhancing CDT. The in vitro and in vivo experimental data indicated that OCZCF could cause remarkable tumour inhibition through enhanced synergetic PDT and CDT.

These works clearly highlight that multifunctional nanoplatforms may open up a new way for engineering highly efficient multimodal therapeutic approaches for clinical tumour treatment.

Last but not least, biosafety is a major concern for the clinical applications of nanostructures. The possible dark toxicity (toxicity before irradiating with light) of the nanostructure for PDT is a key point that has still to be better understood; therefore, more systematic in vitro and in vivo studies for investigating the toxicity of PDT-based nanostructures are mandatory. One critical issue is the difficulty to compare the performance (both toxicity and efficiency) of the reported nanosystems because studies are performed under quite different conditions. In this direction, the development of standardized protocols for characterizing the
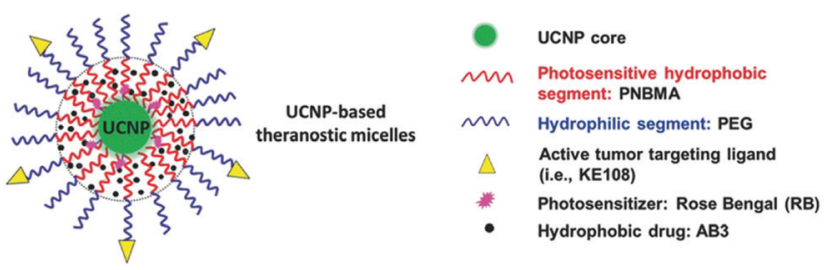

Fig. 10 Multifunctional lanthanide-based UCNPs functionalised with hydrophobic and hydrophilic polymers. The hydrophobic tail contains the PS Rose Bengal and an hydrophobic drug. A targeting ligand is conjugated with the hydrophilic PEG. Under irradiation, both the PS and the drug can be released in a controlled fashion. UCNPs themselves are also used as imaging probes. Taken from ref. 229. Reproduced with permission from John Wiley \& Sons, Copyright(C) 2015 and Copyright(C)2017. relevant features of the nanosystems for PDT would mean a huge step toward their further successful clinical translation. Additionally, to minimize side effects, the nanostructures should be removed from the body after completing their therapeutic functions. Unfortunately, most current NPs used in PDT are non-biodegradable and they may accumulate in the body if their excretion is not completed, which limits their clinical applications. Future research should be directed toward developing biodegradable nanostructures with high phototherapeutic properties.

\section{Conflicts of interest}

There are no conflicts to declare.

\section{Acknowledgements}

The authors want to thank the Sixth Research and Technology Transfer Plan of the University of Seville (VI PPIT-US, to A.E.) and the projects CTQ2016-78580-C2-1-R of the Spanish Ministry of Economy and Competitiveness, COST action CA-18132 "Functional Glyconanomaterials for the Development of Diagnostic and Targeted Therapeutic Probe", and CSIC PIE $202080 \mathrm{E} 157$ (to N. K.). C.C.-C. acknowledges the financial support of the Spanish MINECO through Project No. PID2019-107665RJ-I00 and the Spanish Ministry of Science and Innovation for the financial support under the Ramón $y$ Cajal Program (RYC2019-027527-I).

\section{References}

1 T. J. Dougherty, C. J. Gomer, B. W. Henderson, G. Jori, D. Kessel, M. Korbelik, J. Moan and Q. Peng, Photodynamic Therapy, JNCI, J. Natl. Cancer Inst., 1998, 90, 889-905.

2 M. Hamblin and P. Mroz, Advances in Photodynamic Therapy: Basic, Translational, and Clinical, Artech House, 2008.

$3 \mathrm{M}$. Ochsner, Photophysical and photobiological processes in the photodynamic therapy of tumours, J. Photochem. Photobiol., B, 1997, 39, 1-18.

4 R. W. Redmond and J. N. Gamlin, A Compilation of Singlet Oxygen Yields from Biologically Relevant Molecules, Photochem. Photobiol., 1999, 70, 391-475.

5 E. Gandin, Y. Lion and A. Van, de Vorst, Quantum yield of singlet oxygen production by xanthene derivatives, Photochem. Photobiol., 1983, 37, 271-278.

6 S. T. G. Buck, F. Bettanin, E. Orestes, P. Homem-de-Mello, H. Imasato, R. B. Viana, J. R. Perussi and A. B. F. da Silva, Photodynamic Efficiency of Xanthene Dyes and Their Phototoxicity against a Carcinoma Cell Line: A Computational and Experimental Study, J. Chem., 2017, 2017, 7365263.

7 J. P. Tardivo, A. Del Giglio, C. S. de Oliveira, D. S. Gabrielli, H. C. Junqueira, D. B. Tada, D. Severino, R. de Fátima Turchiello and M. S. Baptista, Methylene blue in 
photodynamic therapy: From basic mechanisms to clinical applications, Photodiagn. Photodyn. Ther., 2005, 2, 175-191.

8 A. Gorman, J. Killoran, C. O'Shea, T. Kenna, W. M. Gallagher and D. F. O'Shea, In Vitro Demonstration of the Heavy-Atom Effect for Photodynamic Therapy, J. Am. Chem. Soc., 2004, 126, 10619-10631.

9 B. Valeur and M. N. Berberan-Santos, Molecular Fluorescence. Principles and Applications, Wiley-VCH, Weinheim, 2013.

10 N. J. Turro, Modern Molecular Photochemistry, University Science Books, Sausalito, CA, 1991.

11 X. Hu, Y.-Y. Huang, Y. Wang, X. Wang and M. R. Hamblin, Antimicrobial Photodynamic Therapy to Control Clinically Relevant Biofilm Infections, Front. Microbiol., 2018, 9, 1299.

12 A. P. Castano, T. N. Demidova and M. R. Hamblin, Mechanisms in photodynamic therapy: part one---photosensitizers, photochemistry and cellular localization, Photodiagn. Photodyn. Ther., 2004, 1, 279-293.

13 J. A. Ibáñez, M. I. Litter and R. A. Pizarro, Photocatalytic bactericidal effect of $\mathrm{TiO}_{2}$ on Enterobacter cloacae: Comparative study with other Gram (-) bacteria, J. Photochem. Photobiol., A, 2003, 157, 81-85.

14 M. Lan, S. Zhao, W. Liu, C.-S. Lee, W. Zhang and P. Wang, Photosensitizers for Photodynamic Therapy, Adv. Healthcare Mater., 2019, 8, 1900132.

15 E. Hemmer, A. Benayas, F. Legare and F. Vetrone, Exploiting the biological windows: current perspectives on fluorescent bioprobes emitting above $1000 \mathrm{~nm}$, Nanoscale Horiz., 2016, 1, 168-184.

16 A. Kamkaew, S. H. Lim, H. B. Lee, L. V. Kiew, L. Y. Chung and K. Burgess, BODIPY dyes in photodynamic therapy, Chem. Soc. Rev., 2013, 42, 77-88.

17 B. Hötzer, I. L. Medintz and N. Hildebrandt, Fluorescence in Nanobiotechnology: Sophisticated Fluorophores for Novel Applications, Small, 2012, 8, 2297-2326.

18 A. Escudero, A. I. Becerro, C. Carrillo-Carrion, N. Núñez, M. V. Zyuzin, M. Laguna, D. González-Mancebo, M. Ocaña and W. J. Parak, Rare earth based nanostructured materials: synthesis, functionalization, properties and bioimaging and biosensing applications, Nanophotonics, 2017, 6, 881-921.

19 B. Pelaz, C. Alexiou, R. A. Alvarez-Puebla, F. Alves, A. M. Andrews, S. Ashraf, L. P. Balogh, L. Ballerini, A. Bestetti, C. Brendel, S. Bosi, M. Carril, W. C. W. Chan, C. Chen, X. Chen, X. Chen, Z. Cheng, D. Cui, J. Du, C. Dullin, A. Escudero, N. Feliu, M. Gao, M. George, Y. Gogotsi, A. Grünweller, Z. Gu, N. J. Halas, N. Hampp, R. K. Hartmann, M. C. Hersam, P. Hunziker, J. Jian, X. Jiang, P. Jungebluth, P. Kadhiresan, K. Kataoka, A. Khademhosseini, J. Kopeček, N. A. Kotov, H. F. Krug, D. S. Lee, C.-M. Lehr, K. W. Leong, X.-J. Liang, M. Ling Lim, L. M. Liz-Marzán, X. Ma, P. Macchiarini, H. Meng, H. Möhwald, P. Mulvaney, A. E. Nel, S. Nie, P. Nordlander, T. Okano, J. Oliveira, T. H. Park, R. M. Penner, M. Prato, V. Puntes, V. M. Rotello,
A. Samarakoon, R. E. Schaak, Y. Shen, S. Sjöqvist, A. G. Skirtach, M. G. Soliman, M. M. Stevens, H.-W. Sung, B. Z. Tang, R. Tietze, B. N. Udugama, J. S. VanEpps, T. Weil, P. S. Weiss, I. Willner, Y. Wu, L. Yang, Z. Yue, Q. Zhang, Q. Zhang, X.-E. Zhang, Y. Zhao, X. Zhou and W. J. Parak, Diverse Applications of Nanomedicine, ACS Nano, 2017, 11, 2313-2381.

20 D. Wöhrle, A. Hirth, T. Bogdahn-Rai, G. Schnurpfeil and M. Shopova, Photodynamic therapy of cancer: Second and third generations of photosensitizers, Russ. Chem. Bull., 1998, 47, 807-816.

21 J. Kou, D. Dou and L. Yang, Porphyrin photosensitizers in photodynamic therapy and its applications, Oncotarget, 2017, 8, 81591-81603.

22 C. Tanielian, C. Schweitzer, R. Mechin and C. Wolff, Quantum yield of singlet oxygen production by monomeric and aggregated forms of hematoporphyrin derivative, Free Radical Biol. Med., 2001, 30, 208-212.

23 S. S. Stylli, M. Howes, L. MacGregor, P. Rajendra and A. H. Kaye, Photodynamic therapy of brain tumours: evaluation of porphyrin uptake versus clinical outcome, J. Clin. Neurosci., 2004, 11, 584-596.

24 B. O. Sun, W. Li and N. Liu, Curative effect of the recent photofrin photodynamic adjuvant treatment on young patients with advanced colorectal cancer, Oncol. Lett., 2016, 11, 2071-2074.

25 H. Gattuso, A. Monari and M. Marazzi, Photophysics of chlorin e6: from one- and two-photon absorption to fluorescence and phosphorescence, RSC Adv., 2017, 7, 10992-10999.

26 A. Juzeniene, Chlorin e6-based photosensitizers for photodynamic therapy and photodiagnosis, Photodiagn. Photodyn. Ther., 2009, 6, 94-96.

27 K. Kasuya, M. Shimazu, M. Suzuki, Y. Kuroiwa, J. Usuda, T. Itoi, A. Tsuchida and T. Aoki, Novel photodynamic therapy against biliary tract carcinoma using mono-L: -aspartyl chlorine e6: basic evaluation for its feasibility and efficacy, J. Hepatobiliary Pancreat. Sci., 2010, 17, 313-321.

28 A. Fernando and J. Gariépy, Coupling Chlorin e6 to the surface of Nanoscale Gas Vesicles strongly enhances their intracellular delivery and photodynamic killing of cancer cells, Sci. Rep., 2020, 10, 2802.

29 A. Petri, D. Yova, E. Alexandratou, M. Kyriazi and M. Rallis, Comparative characterization of the cellular uptake and photodynamic efficiency of Foscan ${ }^{\circledR}$ and Fospeg in a human prostate cancer cell line, Photodiagn. Photodyn. Ther., 2012, 9, 344-354.

30 J. C. Kennedy and R. H. Pottier, New trends in photobiology: Endogenous protoporphyrin IX, a clinically useful photosensitizer for photodynamic therapy, J. Photochem. Photobiol., B, 1992, 14, 275-292.

31 K. Mahmoudi, K. L. Garvey, A. Bouras, G. Cramer, H. Stepp, J. G. Jesu Raj, D. Bozec, T. M. Busch and C. G. Hadjipanayis, 5-aminolevulinic acid photodynamic therapy for the treatment of high-grade gliomas, J. NeuroOncol., 2019, 141, 595-607. 
32 M. Rapp, M. Kamp, H.-J. Steiger and M. Sabel, EndoscopicAssisted Visualization of 5-Aminolevulinic Acid-Induced Fluorescence in Malignant Glioma Surgery: A Technical Note, World Neurosurgery, 2014, 82, e277-e279.

33 R. Díez Valle, J. Slof, J. Galván, C. Arza, C. Romariz and C. Vidal, Observational, retrospective study of the effectiveness of 5-aminolevulinic acid in malignant glioma surgery in Spain (The VISIONA study), Neurología, 2014, 29, 131-138.

34 T. Nakayama, S. Otsuka, T. Kobayashi, H. Okajima, K. Matsumoto, Y. Hagiya, K. Inoue, T. Shuin, M. Nakajima and T. Tanaka, and S.-i. Ogura, Dormant cancer cells accumulate high protoporphyrin IX levels and are sensitive to 5-aminolevulinic acid-based photodynamic therapy, Sci. Rep., 2016, 6, 36478.

35 J. Berlanda, T. Kiesslich, V. Engelhardt, B. Krammer and K. Plaetzer, Comparative in vitro study on the characteristics of different photosensitizers employed in PDT, J. Photochem. Photobiol., B, 2010, 100, 173-180.

36 M. J. Duffy, O. Planas, A. Faust, T. Vogl, S. Hermann, M. Schäfers, S. Nonell and C. A. Strassert, Towards optimized naphthalocyanines as sonochromes for photoacoustic imaging in vivo, Photoacoustics, 2018, 9, 49-61.

37 A. R. Morgan, A. Rampersaud, G. M. Garbo, R. W. Keck and S. H. Selman, New sensitizers for photodynamic therapy. Controlled synthesis of purpurins and their effect on normal tissue, J. Med. Chem., 1989, 32, 904-908.

38 T. Yogo, Y. Urano, Y. Ishitsuka, F. Maniwa and T. Nagano, Highly Efficient and Photostable Photosensitizer Based on BODIPY Chromophore, J. Am. Chem. Soc., 2005, 127, 12162-12163.

39 M. J. Ortiz, A. R. Agarrabeitia, G. Duran-Sampedro, J. Bañuelos Prieto, T. A. Lopez, W. A. Massad, H. A. Montejano, N. A. García and I. Lopez, Arbeloa, Synthesis and functionalization of new polyhalogenated BODIPY dyes. Study of their photophysical properties and singlet oxygen generation, Tetrahedron, 2012, 68, 1153-1162.

40 P. Batat, M. Cantuel, G. Jonusauskas, L. Scarpantonio, A. Palma, D. F. O'Shea and N. D. McClenaghan, BF2Azadipyrromethenes: Probing the Excited-State Dynamics of a NIR Fluorophore and Photodynamic Therapy Agent, J. Phys. Chem. A, 2011, 115, 14034-14039.

41 D. Chen, Z. Zhong, Q. Ma, J. Shao, W. Huang and X. Dong, Aza-BODIPY-Based Nanomedicines in Cancer Phototheranostics, ACS Appl. Mater. Interfaces, 2020, 12, 26914-26925.

42 A. Kamkaew and K. Burgess, Aza-BODIPY dyes with enhanced hydrophilicity, Chem. Commun., 2015, 51, 10664-10667.

43 D. García-Fresnadillo, Y. Georgiadou, G. Orellana, A. M. Braun and E. Oliveros, Singlet-Oxygen $(1 \Delta \mathrm{g})$ Production by Ruthenium(II) complexes containing polyazaheterocyclic ligands in methanol and in water, Helv. Chim. Acta, 1996, 79, 1222-1238.

44 S. Monro, K. L. Colón, H. Yin, J. Roque, P. Konda, S. Gujar, R. P. Thummel, L. Lilge, C. G. Cameron and
S. A. McFarland, Transition Metal Complexes and Photodynamic Therapy from a Tumor-Centered Approach: Challenges, Opportunities, and Highlights from the Development of TLD1433, Chem. Rev., 2019, 119, 797-828.

45 E. Wolcan, Photosensitized generation of singlet oxygen from rhenium(I) complexes: A review, Inorg. Chim. Acta, 2020, 509, 119650.

46 R. T. K. Kwok, C. W. T. Leung, J. W. Y. Lam and B. Z. Tang, Biosensing by luminogens with aggregation-induced emission characteristics, Chem. Soc. Rev., 2015, 44, 4228-4238.

47 J.-J. Hu, W. Jiang, L. Yuan, C. Duan, Q. Yuan, Z. Long, $\mathrm{X}$. Lou and F. Xia, Recent advances in stimuli-responsive theranostic systems with aggregation-induced emission characteristics, Aggregate, 2021, 2, 48-65.

48 J. Liu, J. W. Y. Lam and B. Z. Tang, Aggregation-induced Emission of Silole Molecules and Polymers: Fundamental and Applications, J. Inorg. Organomet. Polym. Mater., 2009, 19, 249.

49 Y. Hong, J. W. Y. Lam and B. Z. Tang, Aggregation-induced emission, Chem. Soc. Rev., 2011, 40, 5361-5388.

50 J. Mei, Y. Hong, J. W. Y. Lam, A. Qin, Y. Tang and B. Z. Tang, Aggregation-Induced Emission: The Whole Is More Brilliant than the Parts, Adv. Mater., 2014, 26, 5429-5479.

$51 \mathrm{~W}$. Wu and Z. Li, Nanoprobes with aggregation-induced emission for theranostics, Mater. Chem. Front., 2021, 5, 603-626.

52 J. Zhang, L. Ning, J. Huang, C. Zhang and K. Pu, Activatable molecular agents for cancer theranostics, Chem. Sci., 2020, 11, 618-630.

53 Y. Yuan, S. Xu, C.-J. Zhang, R. Zhang and B. Liu, Dualtargeted activatable photosensitizers with aggregationinduced emission (AIE) characteristics for image-guided photodynamic cancer cell ablation, J. Mater. Chem. B, 2016, 4, 169-176.

54 W. Wu, D. Mao, S. Xu, S. Ji, F. Hu, D. Ding, D. Kong and B. Liu, High performance photosensitizers with aggregation-induced emission for image-guided photodynamic anticancer therapy, Mater. Horiz., 2017, 4, 1110-1114.

55 W.-T. Li, in Handbook of Biophotonics: Vol. 2: Photonics for Health Care, ed. J. Popp, V. V. Tuchin, A. Chiou and S. H. Heinemann, Wiley, 2011.

56 M. Sivasubramanian, Y. C. Chuang and L.-W. Lo, Evolution of Nanoparticle-Mediated Photodynamic Therapy: From Superficial to Deep-Seated Cancers, Molecules, 2019, 24, 520 .

57 M. R. Hamblin, Fullerenes as photosensitizers in photodynamic therapy: pros and cons, Photochem. Photobiol. Sci., 2018, 17, 1515-1533.

58 Y. Doi, A. Ikeda, M. Akiyama, M. Nagano, T. Shigematsu, T. Ogawa, T. Takeya and T. Nagasaki, Intracellular Uptake and Photodynamic Activity of Water-Soluble [60]- and [70]Fullerenes Incorporated in Liposomes, Chem. - Eur. J., 2008, 14, 8892-8897.

59 W. Zhou, Y. Chen, Y. Zhang, X. Xin, R. Li, C. Xie and Q. Fan, Iodine-Rich Semiconducting Polymer 
Nanoparticles for CT/Fluorescence Dual-Modal ImagingGuided Enhanced Photodynamic Therapy, Small, 2020, 16, 1905641.

60 L. Guo, J. Ge, Q. Liu, Q. Jia, H. Zhang, W. Liu, G. Niu, S. Liu, J. Gong, S. Hackbarth and P. Wang, Versatile Polymer Nanoparticles as Two-Photon-Triggered Photosensitizers for Simultaneous Cellular, Deep-Tissue Imaging, and Photodynamic Therapy, Adv. Healthcare Mater., 2017, 6, 1601431.

61 E. A. Rozhkova, I. Ulasov, B. Lai, N. M. Dimitrijevic, M. S. Lesniak and T. Rajh, A High-Performance Nanobio Photocatalyst for Targeted Brain Cancer Therapy, Nano Lett., 2009, 9, 3337-3342.

62 R. Cai, Y. Kubota, T. Shuin, H. Sakai, K. Hashimoto and A. Fujishima, Induction of Cytotoxicity by Photoexcited TiO2 Particles, Cancer Res., 1992, 52, 2346-2348.

63 S. S. Lucky, N. M. Idris, K. Huang, J. Kim, Z. Li, P. S. P. Thong, R. Xu, K. C. Soo and Y. Zhang, In vivo Biocompatibility, Biodistribution and Therapeutic Efficiency of Titania Coated Upconversion Nanoparticles for Photodynamic Therapy of Solid Oral Cancers, Theranostics, 2016, 6, 1844-1865.

64 S. S. Lucky, N. Muhammad Idris, Z. Li, K. Huang, K. C. Soo and Y. Zhang, Titania Coated Upconversion Nanoparticles for Near-Infrared Light Triggered Photodynamic Therapy, ACS Nano, 2015, 9, 191-205.

65 D. Zhang, L. Wen, R. Huang, H. Wang, X. Hu and D. Xing, Mitochondrial specific photodynamic therapy by rareearth nanoparticles mediated near-infrared graphene quantum dots, Biomaterials, 2018, 153, 14-26.

66 S. Dayal, Y. Lou, A. C. S. Samia, J. C. Berlin, M. E. Kenney and C. Burda, Observation of Non-Förster-Type EnergyTransfer Behavior in Quantum Dot-Phthalocyanine Conjugates, J. Am. Chem. Soc., 2006, 128, 13974-13975.

67 P. Zhang, W. Steelant, M. Kumar and M. Scholfield, Versatile Photosensitizers for Photodynamic Therapy at Infrared Excitation, J. Am. Chem. Soc., 2007, 129, 4526-4527.

68 J. M. Tsay, M. Trzoss, L. Shi, X. Kong, M. Selke, M. E. Jung and S. Weiss, Singlet Oxygen Production by Peptide-Coated Quantum Dot-Photosensitizer Conjugates, J. Am. Chem. Soc., 2007, 129, 6865-6871.

69 W. Sun, T. Shi, L. Luo, X. Chen, P. Lv, Y. Lv, Y. Zhuang, J. Zhu, G. Liu, X. Chen and H. Chen, Monodisperse and Uniform Mesoporous Silicate Nanosensitizers Achieve Low-Dose X-Ray-Induced Deep-Penetrating Photodynamic Therapy, Adv. Mater., 2019, 31, 1808024.

70 Z. Zhu, Z. Tang, J. A. Phillips, R. Yang, H. Wang and W. Tan, Regulation of Singlet Oxygen Generation Using Single-Walled Carbon Nanotubes, J. Am. Chem. Soc., 2008, 130, 10856-10857.

71 L. Tang, X. Yang, Q. Yin, K. Cai, H. Wang, I. Chaudhury, C. Yao, Q. Zhou, M. Kwon, J. A. Hartman, I. T. Dobrucki, L. W. Dobrucki, L. B. Borst, S. Lezmi, W. G. Helferich, A. L. Ferguson, T. M. Fan and J. Cheng, Investigating the optimal size of anticancer nanomedicine, Proc. Natl. Acad. Sci. U. S. A., 2014, 111, 15344-15349.
72 H. Cabral, Y. Matsumoto, K. Mizuno, Q. Chen, M. Murakami, M. Kimura, Y. Terada, M. R. Kano, K. Miyazono, M. Uesaka, N. Nishiyama and K. Kataoka, Accumulation of sub-100 $\mathrm{nm}$ polymeric micelles in poorly permeable tumours depends on size, Nat. Nanotechnol., 2011, 6, 815-823.

73 J.-W. Yoo, D. J. Irvine, D. E. Discher and S. Mitragotri, Bioinspired, bioengineered and biomimetic drug delivery carriers, Nat. Rev. Drug Discovery, 2011, 10, 521-535.

74 M. José Alonso, Nanomedicines for overcoming biological barriers, Biomed. Pharmacother., 2004, 58, 168-172.

75 P. Couvreur, R. Gref, K. Andrieux and C. Malvy, Nanotechnologies for drug delivery: Application to cancer and autoimmune diseases, Prog. Solid State Chem., 2006, 34, 231-235.

76 S. Mura, J. Nicolas and P. Couvreur, Stimuli-responsive nanocarriers for drug delivery, Nat. Mater., 2013, 12, 991-1003.

77 C. C. Berry, in Frontiers of Nanoscience, ed. J. M. de la Fuente and V. Grazu, Elsevier, 2012, vol. 4, pp. 159-180.

78 H. Feracci, B. S. Gutierrez, W. Hempel and I. S. Gil, in Frontiers of Nanoscience, ed. J. M. de la Fuente and V. Grazu, Elsevier, 2012, vol. 4, pp. 197-230.

79 R. Simón-Vázquez, M. Peleteiro, T. Lozano, Á. GonzálezFernández and A. Casal, in Frontiers of Nanoscience, ed. J. M. de la Fuente and V. Grazu, Elsevier, 2012, vol. 4, pp. 443-485.

80 S. Ashraf, B. Pelaz, P. del Pino, M. Carril, A. Escudero, W. J. Parak, M. G. Soliman, Q. Zhang and C. CarrilloCarrion, in Light-Responsive Nanostructured Systems for Applications in Nanomedicine, ed. S. Sortino, Springer International Publishing, Cham, 2016, pp. 169-202, DOI: 10.1007/978-3-319-22942-3_6.

81 R. Vankayala, Y.-K. Huang, P. Kalluru, C.-S. Chiang and K. C. Hwang, First Demonstration of Gold NanorodsMediated Photodynamic Therapeutic Destruction of Tumors via Near Infra-Red Light Activation, Small, 2014, 10, 1612-1622.

82 Ž. Krpetić, P. Nativo, V. Sée, I. A. Prior, M. Brust and M. Volk, Inflicting Controlled Nonthermal Damage to Subcellular Structures by Laser-Activated Gold Nanoparticles, Nano Lett., 2010, 10, 4549-4554.

83 J. Kuncewicz, J. M. Dąbrowski, A. Kyzioł, M. Brindell, P. Łabuz, O. Mazuryk, W. Macyk and G. Stochel, Perspectives of molecular and nanostructured systems with d- and f-block metals in photogeneration of reactive oxygen species for medical strategies, Coord. Chem. Rev., 2019, 398, 113012.

84 P. García Calavia, G. Bruce, L. Pérez-García and D. A. Russell, Photosensitiser-gold nanoparticle conjugates for photodynamic therapy of cancer, Photochem. Photobiol. Sci., 2018, 17, 1534-1552.

85 M. Camerin, M. Moreno, M. J. Marín, C. L. Schofield, I. Chambrier, M. J. Cook, O. Coppellotti, G. Jori and D. A. Russell, Delivery of a hydrophobic phthalocyanine photosensitizer using PEGylated gold nanoparticle 
conjugates for the in vivo photodynamic therapy of amelanotic melanoma, Photochem. Photobiol. Sci., 2016, 15, 618-625.

86 J. Wang, A. M. Potocny, J. Rosenthal and E. S. Day, Gold Nanoshell-Linear Tetrapyrrole Conjugates for Near Infrared-Activated Dual Photodynamic and Photothermal Therapies, ACS Omega, 2020, 5, 926-940.

87 Y. Cheng, J. D. Meyers, A.-M. Broome, M. E. Kenney, J. P. Basilion and C. Burda, Deep Penetration of a PDT Drug into Tumors by Noncovalent Drug-Gold Nanoparticle Conjugates, J. Am. Chem. Soc., 2011, 133, 2583-2591.

88 S. Wang, P. Huang, L. Nie, R. Xing, D. Liu, Z. Wang, J. Lin, S. Chen, G. Niu, G. Lu and X. Chen, Single Continuous Wave Laser Induced Photodynamic/Plasmonic Photothermal Therapy Using Photosensitizer-Functionalized Gold Nanostars, Adv. Mater., 2013, 25, 3055-3061.

89 F. Wu, Y. Liu, Y. Wu, D. Song, J. Qian and B. Zhu, Chlorin e6 and polydopamine modified gold nanoflowers for combined photothermal and photodynamic therapy, J. Mater. Chem. B, 2020, 8, 2128-2138.

90 M. R. Younis, C. Wang, R. An, S. Wang, M. A. Younis, Z.-Q. Li, Y. Wang, A. Ihsan, D. Ye and X.-H. Xia, Low Power Single Laser Activated Synergistic Cancer Phototherapy Using Photosensitizer Functionalized Dual Plasmonic Photothermal Nanoagents, ACS Nano, 2019, 13, 2544-2557.

91 J. Wang, J. Sun, W. Hu, Y. Wang, T. Chou, B. Zhang, Q. Zhang, L. Ren and H. Wang, A Porous Au@Rh Bimetallic Core-Shell Nanostructure as an H2O2-Driven Oxygenerator to Alleviate Tumor Hypoxia for Simultaneous Bimodal Imaging and Enhanced Photodynamic Therapy, Adv. Mater., 2020, 32, 2001862.

92 M. E. Alea-Reyes, J. Soriano, I. Mora-Espí, M. Rodrigues, D. A. Russell, L. Barrios and L. Pérez-García, Amphiphilic gemini pyridinium-mediated incorporation of $\mathrm{Zn}$ (II)mesotetrakis(4-carboxyphenyl)porphyrin into water-soluble gold nanoparticles for photodynamic therapy, Colloids Surf., B, 2017, 158, 602-609.

93 Y. Zhang, K. Aslan, M. J. R. Previte and C. D. Geddes, Plasmonic engineering of singlet oxygen generation, Proc. Natl. Acad. Sci. U. S. A., 2008, 105, 1798-1802.

94 P. García Calavia, M. J. Marín, I. Chambrier, M. J. Cook and D. A. Russell, Towards optimisation of surface enhanced photodynamic therapy of breast cancer cells using gold nanoparticle-photosensitiser conjugates, Photochem. Photobiol. Sci., 2018, 17, 281-289.

95 U. S. Chung, J.-H. Kim, B. Kim, E. Kim, W.-D. Jang and W.-G. Koh, Dendrimer porphyrin-coated gold nanoshells for the synergistic combination of photodynamic and photothermal therapy, Chem. Commun., 2016, 52, 1258-1261.

96 F. Xia, W. Hou, C. Zhang, X. Zhi, J. Cheng, J. M. de la Fuente, J. Song and D. Cui, pH-responsive gold nanoclusters-based nanoprobes for lung cancer targeted near-infrared fluorescence imaging and chemophotodynamic therapy, Acta Bioamater., 2018, 68, 308-319.

97 S. Bayir, A. Barras, R. Boukherroub, S. Szunerits, L. Raehm, S. Richeter and J.-O. Durand, Mesoporous silica nanoparticles in recent photodynamic therapy applications, Photochem. Photobiol. Sci., 2018, 17, 1651-1674.

98 D. Knopp, D. Tang and R. Niessner, Review: Bioanalytical applications of biomolecule-functionalized nanometersized doped silica particles, Anal. Chim. Acta, 2009, 647, 14-30.

99 A. Kamkaew, L. Cheng, S. Goel, H. F. Valdovinos, T. E. Barnhart, Z. Liu and W. Cai, Cerenkov Radiation Induced Photodynamic Therapy Using Chlorin e6-Loaded Hollow Mesoporous Silica Nanoparticles, ACS Appl. Mater. Interfaces, 2016, 8, 26630-26637.

100 W.-H. Chen, G.-F. Luo, W.-X. Qiu, Q. Lei, L.-H. Liu, S.-B. Wang and X.-Z. Zhang, Mesoporous silica-based versatile theranostic nanoplatform constructed by layerby-layer assembly for excellent photodynamic/chemo therapy, Biomaterials, 2017, 117, 54-65.

101 B. Ding, S. Shao, C. Yu, B. Teng, M. Wang, Z. Cheng, K.-L. Wong, P. A. Ma and J. Lin, Large-Pore MesoporousSilica-Coated Upconversion Nanoparticles as Multifunctional Immunoadjuvants with Ultrahigh Photosensitizer and Antigen Loading Efficiency for Improved Cancer Photodynamic Immunotherapy, Adv. Mater., 2018, 30, 1802479.

102 S. Bouramtane, L. Bretin, A. Pinon, D. Leger, B. Liagre, L. Richard, F. Brégier, V. Sol and V. Chaleix, Porphyrinxylan-coated silica nanoparticles for anticancer photodynamic therapy, Carbohydr. Polym., 2019, 213, 168-175.

103 S. Zhang, H. Lv, J. Zhao, M. Cheng and S. Sun, Synthesis of porphyrin-conjugated silica-coated Au nanorods for synergistic photothermal therapy and photodynamic therapy of tumor, Nanotechnology, 2019, 30, 265102.

104 B. Ding, S. Shao, H. Xiao, C. Sun, X. Cai, F. Jiang, X. Zhao, P. A. Ma and J. Lin, $\mathrm{MnFe}_{2} \mathrm{O}_{4}$-decorated large-pore mesoporous silica-coated upconversion nanoparticles for near-infrared light-induced and $\mathrm{O} 2$ self-sufficient photodynamic therapy, Nanoscale, 2019, 11, 14654-14667.

105 C. Gao, Z. Lin, Z. Wu, X. Lin and Q. He, Stem-CellMembrane Camouflaging on Near-Infrared Photoactivated Upconversion Nanoarchitectures for in Vivo RemoteControlled Photodynamic Therapy, ACS Appl. Mater. Interfaces, 2016, 8, 34252-34260.

106 J. Gupta, J. Mohapatra and D. Bahadur, Visible light driven mesoporous Ag-embedded ZnO nanocomposites: reactive oxygen species enhanced photocatalysis, bacterial inhibition and photodynamic therapy, Dalton Trans., 2017, 46, 685-696.

107 X. Zhu, H. Zhou, Y. Liu, Y. Wen, C. Wei, Q. Yu and J. Liu, Transferrin/aptamer conjugated mesoporous ruthenium nanosystem for redox-controlled and targeted chemophotodynamic therapy of glioma, Acta Bioamater., 2018, 82, 143-157.

108 G. S. R. Raju, E. Pavitra, H. Lee, G. Nagaraju, R. Baskaran, S. G. Yang, C. H. Kwak, G. P. Nagaraju, Y. S. Huh and Y.-K. Han, Pre-ouzo effect derived fergusonite gadolinium ortho-niobate mesoporous nanospheroids for multimodal bioimaging and photodynamic therapy, Appl. Surf. Sci., 2020, 505, 144584. 
109 T. Jia, J. Xu, S. Dong, F. He, C. Zhong, G. Yang, H. Bi, M. Xu, Y. Hu, D. Yang, P. Yang and J. Lin, Mesoporous cerium oxide-coated upconversion nanoparticles for tumor-responsive chemo-photodynamic therapy and bioimaging, Chem. Sci., 2019, 10, 8618-8633.

110 A. B. Seabra, in Metal Nanoparticles in Pharma, ed. P. D. M. Rai and P. D. R. Shegokar, Springer International Publishing, Cham, 2017, pp. 3-20, DOI: 10.1007/978-3-319-637907_1.

111 D. Ling, W. Park, S.-J. Park, Y. Lu, K. S. Kim, M. J. Hackett, B. H. Kim, H. Yim, Y. S. Jeon, K. Na and T. Hyeon, Multifunctional Tumor pH-Sensitive Self-Assembled Nanoparticles for Bimodal Imaging and Treatment of Resistant Heterogeneous Tumors, J. Am. Chem. Soc., 2014, 136, 5647-5655.

112 G. Tan, W. Li, J. Cheng, Z. Wang, S. Wei, Y. Jin, C. Guo and F. Qu, Magnetic iron oxide modified pyropheophorbide-a fluorescence nanoparticles as photosensitizers for photodynamic therapy against ovarian cancer (SKOV-3) cells, Photochem. Photobiol. Sci., 2016, 15, 1567-1578.

113 O. Penon, M. J. Marín, D. B. Amabilino, D. A. Russell and L. Pérez-García, Iron oxide nanoparticles functionalized with novel hydrophobic and hydrophilic porphyrins as potential agents for photodynamic therapy, J. Colloid Interface Sci., 2016, 462, 154-165.

114 A. Amirshaghaghi, L. Yan, J. Miller, Y. Daniel, J. M. Stein, T. M. Busch, Z. Cheng and A. Tsourkas, Chlorin e6-Coated Superparamagnetic Iron Oxide Nanoparticle (SPION) Nanoclusters as a Theranostic Agent for Dual-Mode Imaging and Photodynamic Therapy, Sci. Rep., 2019, 9, 2613.

115 R. Di Corato, G. Béalle, J. Kolosnjaj-Tabi, A. Espinosa, O. Clément, A. K. A. Silva, C. Ménager and C. Wilhelm, Combining Magnetic Hyperthermia and Photodynamic Therapy for Tumor Ablation with Photoresponsive Magnetic Liposomes, ACS Nano, 2015, 9, 2904-2916.

116 L. Zeng, L. Luo, Y. Pan, S. Luo, G. Lu and A. Wu, In vivo targeted magnetic resonance imaging and visualized photodynamic therapy in deep-tissue cancers using folic acid-functionalized superparamagnetic-upconversion nanocomposites, Nanoscale, 2015, 7, 8946-8954.

117 M. V. Zyuzin, A. S. Timin and G. B. Sukhorukov, Multilayer Capsules Inside Biological Systems: State-of-the-Art and Open Challenges, Langmuir, 2019, 35, 4747-4762.

118 A. V. Ermakov, R. A. Verkhovskii, I. V. Babushkina, D. B. Trushina, O. A. Inozemtseva, E. A. Lukyanets, V. J. Ulyanov, D. A. Gorin, S. Belyakov and M. N. Antipina, In Vitro Bioeffects of Polyelectrolyte Multilayer Microcapsules Post-Loaded with Water-Soluble Cationic Photosensitizer, Pharmaceutics, 2020, 12, 610.

119 A. R. Simioni, P. C. C. de Jesus and A. C. Tedesco, Layer-bylayer hollow photosensitizer microcapsule design via a manganese carbonate hard template for photodynamic therapy in cells, Photodiagn. Photodyn. Ther., 2018, 22, 169-177.

120 Q. Dong, J. Li, L. Cui, H. Jian, A. Wang and S. Bai, Using porous $\mathrm{CaCO} 3 /$ hyaluronic acid nanocages to accommodate hydrophobic photosensitizer in aqueous media for photodynamic therapy, Colloids Surf., A, 2017, 516, 190-198.

121 N. A. N. Hanafy, M. El-Kemary and S. Leporatti, Micelles Structure Development as a Strategy to Improve Smart Cancer Therapy, Cancers, 2018, 10, 238.

122 A. Escudero, S. Carregal-Romero, A. B. Miguel-Coello and J. Ruíz-Cabello, in Frontiers of Nanoscience, ed. W. J. Parak and N. Feliu, Elsevier, 2020, vol. 16, pp. 201-232.

123 T. F. A. De Greef, M. M. J. Smulders, M. Wolffs, A. P. H. J. Schenning, R. P. Sijbesma and E. W. Meijer, Supramolecular Polymerization, Chem. Rev., 2009, 109, 5687-5754.

124 R. Dong, Y. Pang, Y. Su and X. Zhu, Supramolecular hydrogels: synthesis, properties and their biomedical applications, Biomater. Sci., 2015, 3, 937-954.

125 T. Zinn, L. Willner, R. Lund, V. Pipich, M.-S. Appavou and D. Richter, Surfactant or block copolymer micelles? Structural properties of a series of well-defined n-alkyl-PEO micelles in water studied by SANS, Soft Matter, 2014, 10, 5212-5220.

126 S. H. Kang, B. M. Jung, W. J. Kim and J. Y. Chang, Embedding Nanofibers in a Polymer Matrix by Polymerization of Organogels Comprising Heterobifunctional Organogelators and Monomeric Solvents, Chem. Mater., 2008, 20, 5532-5540.

127 E. Gravel, J. Ogier, T. Arnauld, N. Mackiewicz, F. Ducongé and E. Doris, Drug Delivery and Imaging with Polydiacetylene Micelles, Chem. - Eur. J., 2012, 18, 400-408.

128 R. W. Carpick, D. Y. Sasaki, M. S. Marcus, M. A. Eriksson and A. R. Burns, Polydiacetylene films: a review of recent investigations into chromogenic transitions and nanomechanical properties, J. Phys.: Condens. Matter, 2004, 16, R679-R697.

129 M. Assali, J.-J. Cid, I. Fernández and N. Khiar, Supramolecular Diversity through Click Chemistry: Switching from Nanomicelles to 1D-Nanotubes and Tridimensional Hydrogels, Chem. Mater., 2013, 25, 4250-4261.

130 Y. Lu, E. Zhang, J. Yang and Z. Cao, Strategies to improve micelle stability for drug delivery, Nano Res., 2018, 11, 4985-4998.

131 P. Avci, S. S. Erdem and M. R. Hamblin, Photodynamic Therapy: One Step Ahead with Self-Assembled Nanoparticles, J. Biomed. Nanotechnol., 2014, 10, 1937-1952.

132 C. F. van Nostrum, Polymeric micelles to deliver photosensitizers for photodynamic therapy, Adv. Drug Delivery Rev., 2004, 56, 9-16.

133 Y. Xue, J. Tian, Z. Liu, J. Chen, M. Wu, Y. Shen and W. Zhang, A Redox Stimulation-Activated Amphiphile for Enhanced Photodynamic Therapy, Biomacromolecules, 2019, 20, 2796-2808.

134 H. Cabral, K. Miyata, K. Osada and K. Kataoka, Block Copolymer Micelles in Nanomedicine Applications, Chem. Rev., 2018, 118, 6844-6892.

135 J. Zhao, X. Zhang, L. Fang, C. Gao, C. Xu and S. Gou, Iridium(III) Complex-Derived Polymeric Micelles with Low Dark Toxicity and Strong NIR Excitation for Phototherapy and Chemotherapy, Small, 2020, 16, 2000363. 
136 N. Nishiyama, Y. Morimoto, W.-D. Jang and K. Kataoka, Design and development of dendrimer photosensitizerincorporated polymeric micelles for enhanced photodynamic therapy, Adv. Drug Delivery Rev., 2009, 61, 327-338.

137 J. Tian, L. Xu, Y. Xue, X. Jiang and W. Zhang, Enhancing Photochemical Internalization of DOX through a Porphyrin-based Amphiphilic Block Copolymer, Biomacromolecules, 2017, 18, 3992-4001.

138 S. Su, Y. Ding, Y. Li, Y. Wu and G. Nie, Integration of photothermal therapy and synergistic chemotherapy by a porphyrin self-assembled micelle confers chemosensitivity in triple-negative breast cancer, Biomaterials, 2016, 80, 169-178.

139 D.-Q. Wu, Z.-Y. Li, C. Li, J.-J. Fan, B. Lu, C. Chang, S.X. Cheng, X.-Z. Zhang and R.-X. Zhuo, Porphyrin and Galactosyl Conjugated Micelles for Targeting Photodynamic Therapy, Pharm. Res., 2009, 27, 187.

140 X. Liu, G. Yang, L. Zhang, Z. Liu, Z. Cheng and X. Zhu, Photosensitizer cross-linked nano-micelle platform for multimodal imaging guided synergistic photothermal/ photodynamic therapy, Nanoscale, 2016, 8, 15323-15339.

141 H.-H. Han, C.-Z. Wang, Y. Zang, J. Li, T. D. James and X.-P. He, Supramolecular core-glycoshell polythiophene nanodots for targeted imaging and photodynamic therapy, Chem. Commun., 2017, 53, 9793-9796.

142 V. Khatri, S. Bhatia, K. Achazi, S. Deep, E. Kohli, S. K. Sharma, R. Haag and A. K. Prasad, Lipase-mediated synthesis of sugar-PEG-based amphiphiles for encapsulation and stabilization of indocyanine green, $R S C A d v$., 2017, 7, 37534-37541.

143 C. Pais-Silva, D. de Melo-Diogo and I. J. Correia, IR780loaded TPGS-TOS micelles for breast cancer photodynamic therapy, Eur. J. Pharm. Biopharm., 2017, 113, 108-117.

144 D. S. Pellosi, I. R. Calori, L. B. de Paula, N. Hioka, F. Quaglia and A. C. Tedesco, Multifunctional theranostic Pluronic mixed micelles improve targeted photoactivity of Verteporfin in cancer cells, Mater. Sci. Eng., C, 2017, 71, 1-9.

145 Y. Fan and Q. Zhang, Development of liposomal formulations: From concept to clinical investigations, Asian J. Pharm. Sci., 2013, 8, 81-87.

146 M. Rahman, S. Beg, A. Verma, F. Anwar, A. Samad and V. Kumar, in Nanotechnology-Based Approaches for Targeting and Delivery of Drugs and Genes, ed. V. Mishra, P. Kesharwani, M. C. I. Mohd Amin and A. Iyer, Academic Press, 2017, pp. 151-166, DOI: 10.1016/B978-0-12-8097175.00005-1.

147 E. Paszko, C. Ehrhardt, M. O. Senge, D. P. Kelleher and J. V. Reynolds, Nanodrug applications in photodynamic therapy, Photodiagn. Photodyn. Ther., 2011, 8, 14-29.

148 P. Skupin-Mrugalska, J. Piskorz, T. Goslinski, J. Mielcarek, K. Konopka and N. Düzgüneş, Current status of liposomal porphyrinoid photosensitizers, Drug Discovery Today, 2013, 18, 776-784.

149 H. Fukuda, S. Paredes and A. M. del C. Battle, Tumourlocalizing properties of porphyrins in vivo studies using free and liposome encapsulated aminolevulinic acid, Comp. Biochem. Physiol., Part B: Biochem. Mol. Biol., 1992, 102, 433-436.

150 E. Gross, B. Ehrenberg and F. M. Johnson, Singlet Oxygen Generation By Porphyrins and the Kinetics of 9,10Dimethylanthracene Photosensitization in Liposomes, Photochem. Photobiol., 1993, 57, 808-813.

151 F. Jiang, L. Lilge, J. Grenier, Y. Li, M. D. Wilson and M. Chopp, Photodynamic therapy of U87 human glioma in nude rat using liposome-delivered photofrin, Lasers Surg. Med., 1998, 22, 74-80.

152 A. Akbarzadeh, R. Rezaei-Sadabady, S. Davaran, S. W. Joo, N. Zarghami, Y. Hanifehpour, M. Samiei, M. Kouhi and K. Nejati-Koshki, Liposome: classification, preparation, and applications, Nanoscale Res. Lett., 2013, 8, 102.

153 E. Temizel, T. Sagir, E. Ayan, S. Isik and R. Ozturk, Delivery of lipophilic porphyrin by liposome vehicles: Preparation and photodynamic therapy activity against cancer cell lines, Photodiagn. Photodyn. Ther., 2014, 11, 537-545.

154 T. Gayathri, A. Vijayalakshmi, S. Mangalath, J. Joseph, N. M. Rao and S. P. Singh, Study on Liposomal Encapsulation of New Bodipy Sensitizers for Photodynamic Therapy, ACS Med. Chem. Lett., 2018, 9, 323-327.

155 Y.-P. Fang, Y.-H. Tsai, P.-C. Wu and Y.-B. Huang, Comparison of 5-aminolevulinic acid-encapsulated liposome versus ethosome for skin delivery for photodynamic therapy, Int. J. Pharm., 2008, 356, 144-152.

156 P.-T. Wu, C.-L. Lin, C.-W. Lin, N.-C. Chang, W.-B. Tsai and J. Yu, Methylene-Blue-Encapsulated Liposomes as Photodynamic Therapy Nano Agents for Breast Cancer Cells, Nanomaterials, 2018, 9, 14.

157 S. Sur, A. C. Fries, K. W. Kinzler, S. Zhou and B. Vogelstein, Remote loading of preencapsulated drugs into stealth liposomes, Proc. Natl. Acad. Sci. U. S. A., 2014, 111, 2283-2288.

158 A. Ikeda, S. Hino, T. Mae, Y. Tsuchiya, K. Sugikawa, M. Tsukamoto, K. Yasuhara, H. Shigeto, H. Funabashi, A. Kuroda and M. Akiyama, Porphyrin-uptake in liposomes and living cells using an exchange method with cyclodextrin, RSC Adv., 2015, 5, 105279-105287.

159 J. G. Christie and U. B. Kompella, Ophthalmic light sensitive nanocarrier systems, Drug Discovery Today, 2008, 13, 124-134.

160 V. Reshetov, D. Kachatkou, T. Shmigol, V. Zorin, M.-A. D'Hallewin, F. Guillemin and L. Bezdetnaya, Redistribution of meta-tetra(hydroxyphenyl)chlorin (m-THPC) from conventional and PEGylated liposomes to biological substrates, Photochem. Photobiol. Sci., 2011, 10, 911-919.

161 J. Kuntsche, I. Freisleben, F. Steiniger and A. Fahr, Temoporfin-loaded liposomes: Physicochemical characterization, Eur. J. Pharm. Sci., 2010, 40, 305-315.

162 E. Gaio, D. Scheglmann, E. Reddi and F. Moret, Uptake and photo-toxicity of Foscan ${ }^{\circledR}$, Foslip ${ }^{\circledR}$ and Fospeg ${ }^{\circledR}$ in multicellular tumor spheroids, J. Photochem. Photobiol., B, 2016, 161, 244-252.

163 M. Millard, I. Yakavets, M. Piffoux, A. Brun, F. Gazeau, J.-M. Guigner, J. Jasniewski, H.-P. Lassalle, C. Wilhelm and 
L. Bezdetnaya, mTHPC-loaded extracellular vesicles outperform liposomal and free mTHPC formulations by an increased stability, drug delivery efficiency and cytotoxic effect in tridimensional model of tumors, Drug Delivery, 2018, 25, 1790-1801.

164 H. T. Shin, J. H. Kim, J. Shim, J. H. Lee, D. Y. Lee, J. H. Lee and J. M. Yang, Photodynamic therapy using a new formulation of 5-aminolevulinic acid for wrinkles in Asian skin: A randomized controlled split face study, J. Dermatol. Treat., 2015, 26, 246-251.

165 M. Sadasivam, P. Avci, G. K. Gupta, S. Lakshmanan, R. Chandran, Y.-Y. Huang, R. Kumar and M. R. Hamblin, Self-assembled liposomal nanoparticles in photodynamic therapy, Eur. J. Nanomed., 2013, 5, 115-129.

166 N. Gross, M. Ranjbar, C. Evers, J. Hua, G. Martin, B. Schulze, U. Michaelis, L. L. Hansen and H. T. Agostini, Choroidal neovascularization reduced by targeted drug delivery with cationic liposomeencapsulated paclitaxel or targeted photodynamic therapy with verteporfin encapsulated in cationic liposomes, Mol. Vis., 2013, 19, 54-61.

167 H. Zhou, L. Xia, J. Zhong, S. Xiong, X. Yi, L. Chen, R. Zhu, Q. Shi and K. Yang, Plant-derived chlorophyll derivative loaded liposomes for tri-model imaging guided photodynamic therapy, Nanoscale, 2019, 11, 19823-19831.

168 X. Song, L. Feng, C. Liang, M. Gao, G. Song and Z. Liu, Liposomes co-loaded with metformin and chlorin e6 modulate tumor hypoxia during enhanced photodynamic therapy, Nano Res., 2017, 10, 1200-1212.

169 L. Feng, L. Cheng, Z. Dong, D. Tao, T. E. Barnhart, W. Cai, M. Chen and Z. Liu, Theranostic Liposomes with HypoxiaActivated Prodrug to Effectively Destruct Hypoxic Tumors Post-Photodynamic Therapy, ACS Nano, 2017, 11, 927-937.

170 Y. Yang, L. Wang, H. Cao, Q. Li, Y. Li, M. Han, H. Wang and J. Li, Photodynamic Therapy with Liposomes Encapsulating Photosensitizers with Aggregation-Induced Emission, Nano Lett., 2019, 19, 1821-1826.

171 M. Giménez-Marqués, T. Hidalgo, C. Serre and P. Horcajada, Nanostructured metal-organic frameworks and their bio-related applications, Coord. Chem. Rev., 2016, 307, 342-360.

172 C. He, D. Liu and W. Lin, Nanomedicine Applications of Hybrid Nanomaterials Built from Metal-Ligand Coordination Bonds: Nanoscale Metal-Organic Frameworks and Nanoscale Coordination Polymers, Chem. Rev., 2015, 115, 11079-11108.

173 H.-C. J. Zhou and S. Kitagawa, Metal-Organic Frameworks (MOFs), Chem. Soc. Rev., 2014, 43, 5415-5418.

174 H. Furukawa, K. E. Cordova, M. O’Keeffe and O. M. Yaghi, The Chemistry and Applications of Metal-Organic Frameworks, Science, 2013, 341, 1230444.

175 G. Maurin, C. Serre, A. Cooper and G. Férey, The new age of MOFs and of their porous-related solids, Chem. Soc. Rev., 2017, 46, 3104-3107.

176 N. Stock and S. Biswas, Synthesis of Metal-Organic Frameworks (MOFs): Routes to Various MOF Topologies,
Morphologies, and Composites, Chem. Rev., 2012, 112, 933-969.

177 G. Férey and C. Serre, Large breathing effects in threedimensional porous hybrid matter: facts, analyses, rules and consequences, Chem. Soc. Rev., 2009, 38, 1380-1399.

178 C. Carrillo-Carrión, Nanoscale metal-organic frameworks as key players in the context of drug delivery: evolution toward theranostic platforms, Anal. Bioanal. Chem., 2020, 412, 37-54.

179 P. Horcajada, T. Chalati, C. Serre, B. Gillet, C. Sebrie, T. Baati, J. F. Eubank, D. Heurtaux, P. Clayette, C. Kreuz, J.-S. Chang, Y. K. Hwang, V. Marsaud, P.-N. Bories, L. Cynober, S. Gil, G. Férey, P. Couvreur and R. Gref, Porous metal-organic-framework nanoscale carriers as a potential platform for drug delivery and imaging, Nat. Mater., 2010, 9, 172-178.

180 G. Lan, K. Ni and W. Lin, Nanoscale metal-organic frameworks for phototherapy of cancer, Coord. Chem. Rev., 2019, 379, 65-81.

181 M. Lismont, L. Dreesen and S. Wuttke, Metal-Organic Framework Nanoparticles in Photodynamic Therapy: Current Status and Perspectives, Adv. Funct. Mater., 2017, 27, 1606314.

182 J. Park, Q. Jiang, D. Feng, L. Mao and H.-C. Zhou, SizeControlled Synthesis of Porphyrinic Metal-Organic Framework and Functionalization for Targeted Photodynamic Therapy, J. Am. Chem. Soc., 2016, 138, 3518-3525.

183 W. Wang, L. Wang, Z. Li and Z. Xie, BODIPY-containing nanoscale metal-organic frameworks for photodynamic therapy, Chem. Commun., 2016, 52, 5402-5405.

184 Z. Ruan, Y. Zhao, P. Yuan, L. Liu, Y. Wang and L. Yan, PEG conjugated BODIPY-Br2 as macro-photosensitizer for efficient imaging-guided photodynamic therapy, J. Mater. Chem. B, 2018, 6, 753-762.

185 X. Li, L. Lachmanski, S. Safi, S. Sene, C. Serre, J. M. Grenèche, J. Zhang and R. Gref, New insights into the degradation mechanism of metal-organic frameworks drug carriers, Sci. Rep., 2017, 7, 13142.

186 W. Lin, W. J. Rieter and K. M. L. Taylor, Modular Synthesis of Functional Nanoscale Coordination Polymers, Angew. Chem., Int. Ed., 2009, 48, 650-658.

187 H. Ren, L. Zhang, J. An, T. Wang, L. Li, X. Si, L. He, X. Wu, C. Wang and Z. Su, Polyacrylic acid@zeolitic imidazolate framework-8 nanoparticles with ultrahigh drug loading capability for pH-sensitive drug release, Chem. Commun., 2014, 50, 1000-1002.

188 B. Lei, M. Wang, Z. Jiang, W. Qi, R. Su and Z. He, Constructing Redox-Responsive Metal-Organic Framework Nanocarriers for Anticancer Drug Delivery, ACS Appl. Mater. Interfaces, 2018, 10, 16698-16706.

189 C. Carrillo-Carrión, R. Martínez, M. F. Navarro Poupard, B. Pelaz, E. Polo, A. Arenas-Vivo, A. Olgiati, P. Taboada, M. G. Soliman, Ú. Catalán, S. Fernández-Castillejo, R. Solà, W. J. Parak, P. Horcajada, R. A. Alvarez-Puebla and P. del Pino, Aqueous Stable Gold Nanostar/ZIF-8 Nanocomposites for Light-Triggered Release of Active Cargo 
Inside Living Cells, Angew. Chem., Int. Ed., 2019, 58, 7078-7082.

190 K. Lu, C. He and W. Lin, Nanoscale Metal-Organic Framework for Highly Effective Photodynamic Therapy of Resistant Head and Neck Cancer, J. Am. Chem. Soc., 2014, 136, 16712-16715.

191 F. Hu, D. Mao, Kenry, Y. Wang, W. Wu, D. Zhao, D. Kong and B. Liu, Metal-Organic Framework as a Simple and General Inert Nanocarrier for Photosensitizers to Implement Activatable Photodynamic Therapy, Adv. Funct. Mater., 2018, 28, 1707519.

192 D. Xu, Y. You, F. Zeng, Y. Wang, C. Liang, H. Feng and X. Ma, Disassembly of Hydrophobic Photosensitizer by Biodegradable Zeolitic Imidazolate Framework-8 for Photodynamic Cancer Therapy, ACS Appl. Mater. Interfaces, 2018, 10, 15517-15523.

193 S. S. Lucky, K. C. Soo and Y. Zhang, Nanoparticles in Photodynamic Therapy, Chem. Rev., 2015, 115, 1990-2042.

194 J.-N. Liu, W. Bu and J. Shi, Chemical Design and Synthesis of Functionalized Probes for Imaging and Treating Tumor Hypoxia, Chem. Rev., 2017, 117, 6160-6224.

195 X. Tong, A. Srivatsan, O. Jacobson, Y. Wang, Z. Wang, X. Yang, G. Niu, D. O. Kiesewetter, H. Zheng and X. Chen, Monitoring Tumor Hypoxia Using 18F-FMISO PET and Pharmacokinetics Modeling after Photodynamic Therapy, Sci. Rep., 2016, 6, 31551.

196 W. Fan, P. Huang and X. Chen, Overcoming the Achilles' heel of photodynamic therapy, Chem. Soc. Rev., 2016, 45, 6488-6519.

197 G. Lan, K. Ni, Z. Xu, S. S. Veroneau, Y. Song and W. Lin, Nanoscale Metal-Organic Framework Overcomes Hypoxia for Photodynamic Therapy Primed Cancer Immunotherapy, J. Am. Chem. Soc., 2018, 140, 5670-5673.

198 Y. Zhang, F. Wang, C. Liu, Z. Wang, L. Kang, Y. Huang, K. Dong, J. Ren and X. Qu, Nanozyme Decorated MetalOrganic Frameworks for Enhanced Photodynamic Therapy, ACS Nano, 2018, 12, 651-661.

199 Z. Xie, S. Liang, X. Cai, B. Ding, S. Huang, Z. Hou, P. A. Ma, Z. Cheng and J. Lin, O2-Cu/ZIF-8@Ce6/ZIF-8@F127 Composite as a Tumor Microenvironment-Responsive Nanoplatform with Enhanced Photo-/Chemodynamic Antitumor Efficacy, ACS Appl. Mater. Interfaces, 2019, 11, 31671-31680.

200 Z. Xie, X. Cai, C. Sun, S. Liang, S. Shao, S. Huang, Z. Cheng, M. Pang, B. Xing, A. A. A. Kheraif and J. Lin, O2-Loaded pHResponsive Multifunctional Nanodrug Carrier for Overcoming Hypoxia and Highly Efficient Chemo-Photodynamic Cancer Therapy, Chem. Mater., 2019, 31, 483-490.

201 G. Lan, K. Ni, S. S. Veroneau, X. Feng, G. T. Nash, T. Luo, Z. $\mathrm{Xu}$ and $\mathrm{W}$. Lin, Titanium-Based Nanoscale MetalOrganic Framework for Type I Photodynamic Therapy, J. Am. Chem. Soc., 2019, 141, 4204-4208.

202 X. Cai, Z. Xie, B. Ding, S. Shao, S. Liang, M. Pang and J. Lin, Monodispersed copper(I)-Based Nano Metal-Organic Framework as a Biodegradable Drug Carrier with Enhanced Photodynamic Therapy Efficacy, Adv. Sci., 2019, 6, 1900848.
203 Y. Li, Z. Di, J. Gao, P. Cheng, C. Di, G. Zhang, B. Liu, X. Shi, L.-D. Sun, L. Li and C.-H. Yan, Heterodimers Made of Upconversion Nanoparticles and Metal-Organic Frameworks, J. Am. Chem. Soc., 2017, 139, 13804-13810.

204 K. Lu, C. He, N. Guo, C. Chan, K. Ni, G. Lan, H. Tang, C. Pelizzari, Y.-X. Fu, M. T. Spiotto, R. R. Weichselbaum and W. Lin, Low-dose X-ray radiotherapy-radiodynamic therapy via nanoscale metal-organic frameworks enhances checkpoint blockade immunotherapy, Nat. Biomed. Eng., 2018, 2, 600-610.

205 Y. Nakamura, A. Mochida, P. L. Choyke and H. Kobayashi, Nanodrug Delivery: Is the Enhanced Permeability and Retention Effect Sufficient for Curing Cancer?, Bioconjugate Chem., 2016, 27, 2225-2238.

206 M. F. Attia, N. Anton, J. Wallyn, Z. Omran and T. F. Vandamme, An overview of active and passive targeting strategies to improve the nanocarriers efficiency to tumour sites, J. Pharm. Pharmacol., 2019, 71, 1185-1198.

207 S. A. Chew, S. Moscato, S. George, B. Azimi and S. Danti, Liver Cancer: Current and Future Trends Using Biomaterials, Cancers, 2019, 11, 2026.

208 A. Cimadamore, M. Cheng, M. Santoni, A. Lopez-Beltran, N. Battelli, F. Massari, A. B. Galosi, M. Scarpelli and R. Montironi, New Prostate Cancer Targets for Diagnosis, Imaging, and Therapy: Focus on Prostate-Specific Membrane Antigen, Front. Oncol., 2018, 8, 653.

209 D. B. Werz and P. H. Seeberger, Carbohydrates as the Next Frontier in Pharmaceutical Research, Chem. - Eur. J., 2005, 11, 3194-3206.

210 N. Kottari, Y. M. Chabre, R. Sharma and R. Roy, in Multifaceted Development and Application of Biopolymers for Biology, Biomedicine and Nanotechnology, ed. P. K. Dutta and J. Dutta, Springer Berlin Heidelberg, Berlin, Heidelberg, 2013, pp. 297-341, DOI: 10.1007/12_2012_208.

211 S. Gim, Y. Zhu, P. H. Seeberger and M. Delbianco, Carbohydrate-based nanomaterials for biomedical applications, Wiley Interdiscip. Rev.: Nanomed. Nanobiotechnol., 2019, 11, e1558.

212 O. Vaillant, K. E. Cheikh, D. Warther, D. Brevet, M. Maynadier, E. Bouffard, F. Salgues, A. Jeanjean, P. Puche, C. Mazerolles, P. Maillard, O. Mongin, M. Blanchard-Desce, L. Raehm, X. Rébillard, J.-O. Durand, M. Gary-Bobo, A. Morère and M. Garcia, Mannose-6Phosphate Receptor: A Target for Theranostics of Prostate Cancer, Angew. Chem., Int. Ed., 2015, 54, 5952-5956.

213 S. H. Medina, V. Tekumalla, M. V. Chevliakov, D. S. Shewach, W. D. Ensminger and M. E. H. El-Sayed, $\mathrm{N}$-acetylgalactosamine-functionalized dendrimers as hepatic cancer cell-targeted carriers, Biomaterials, 2011, 32, 4118-4129.

214 J. Lu, J. Wang and D. Ling, Surface Engineering of Nanoparticles for Targeted Delivery to Hepatocellular Carcinoma, Small, 2018, 14, 1702037.

215 A. A. D'Souza and P. V. Devarajan, Asialoglycoprotein receptor mediated hepatocyte targeting - Strategies and applications, J. Controlled Release, 2015, 203, 126-139. 
216 B. Marie, M. Bohuslav and M.-D. Beatrice, Folate receptor: a potential target in ovarian cancer, Pteridines, 2015, 26, 1.

217 S. Lütje, R. Slavik, W. Fendler, K. Herrmann and M. Eiber, PSMA ligands in prostate cancer - Probe optimization and theranostic applications, Methods, 2017, 130, 42-50.

218 L. Liu, Z. Ruan, T. Li, P. Yuan and L. Yan, Near infrared imaging-guided photodynamic therapy under an extremely low energy of light by galactose targeted amphiphilic polypeptide micelle encapsulating BODIPY-Br2, Biomater. Sci., 2016, 4, 1638-1645.

219 Y. Wei, F. Zhou, D. Zhang, Q. Chen and D. Xing, A graphene oxide based smart drug delivery system for tumor mitochondria-targeting photodynamic therapy, Nanoscale, 2016, 8, 3530-3538.

220 J. Xu, F. Zeng, H. Wu, C. Yu and S. Wu, Dual-Targeting Nanosystem for Enhancing Photodynamic Therapy Efficiency, ACS Appl. Mater. Interfaces, 2015, 7, 9287-9296.

221 J. D. Meyers, Y. Cheng, A.-M. Broome, R. S. Agnes, M. D. Schluchter, S. Margevicius, X. Wang, M. E. Kenney, C. Burda and J. P. Basilion, Peptide-Targeted Gold Nanoparticles for Photodynamic Therapy of Brain Cancer, Part. Part. Syst. Charact., 2015, 32, 448-457.

222 L. Liang, A. Care, R. Zhang, Y. Lu, N. H. Packer, A. Sunna, Y. Qian and A. V. Zvyagin, Facile Assembly of Functional Upconversion Nanoparticles for Targeted Cancer Imaging and Photodynamic Therapy, ACS Appl. Mater. Interfaces, 2016, 8, 11945-11953.

223 S. E. Weinberg and N. S. Chandel, Targeting mitochondria metabolism for cancer therapy, Nat. Chem. Biol., 2015, 11, 9-15.
224 Z. Cao, D. Li, J. Wang, M. Xiong and X. Yang, Direct Nucleus-Targeted Drug Delivery Using Cascade pHe/Photo Dual-Sensitive Polymeric Nanocarrier for Cancer Therapy, Small, 2019, 15, 1902022.

225 K. Laws, G. Bineva-Todd, A. Eskandari, C. Lu, N. O’Reilly and K. Suntharalingam, A Copper(II) Phenanthroline Metallopeptide That Targets and Disrupts Mitochondrial Function in Breast Cancer Stem Cells, Angew. Chem., Int. Ed., 2018, 57, 287-291.

226 H. Cheng, R.-R. Zheng, G.-L. Fan, J.-H. Fan, L.-P. Zhao, X.-Y. Jiang, B. Yang, X.-Y. Yu, S.-Y. Li and X.-Z. Zhang, Mitochondria and plasma membrane dual-targeted chimeric peptide for single-agent synergistic photodynamic therapy, Biomaterials, 2019, 188, 1-11.

227 P. Zhang, H. Huang, S. Banerjee, G. J. Clarkson, C. Ge, C. Imberti and P. J. Sadler, Nucleus-Targeted Organoiridium-Albumin Conjugate for Photodynamic Cancer Therapy, Angew. Chem., Int. Ed., 2019, 58, 2350-2354.

228 X. Yi, J. Dai, Y. Han, M. Xu, X. Zhang, S. Zhen, Z. Zhao, X. Lou and F. Xia, A high therapeutic efficacy of polymeric prodrug nano-assembly for a combination of photodynamic therapy and chemotherapy, Commun. Biol., 2018, 1, 202.

229 G. Chen, R. Jaskula-Sztul, C. R. Esquibel, I. Lou, Q. Zheng, A. Dammalapati, A. Harrison, K. W. Eliceiri, W. Tang, H. Chen and S. Gong, Neuroendocrine Tumor-Targeted Upconversion Nanoparticle-Based Micelles for Simultaneous NIR-Controlled Combination Chemotherapy and Photodynamic Therapy, and Fluorescence Imaging, $A d v$. Funct. Mater., 2017, 27, 1604671. 